\title{
Macroscopic rheological behavior of suspensions of soft solid particles in yield stress fluids
}

\author{
Reza Avazmohammadi ${ }^{\mathrm{a}, \mathrm{b}, *}$, Pedro Ponte Castañeda ${ }^{\mathrm{a}}$ \\ ${ }^{a}$ Department of Mechanical Engineering and Applied Mechanics, University of Pennsylvania, \\ Philadelphia, PA 19104, U.S.A. \\ ${ }^{b}$ Institute for Computational Engineering and Sciences, University of Texas at Austin, TX 78712, \\ U.S.A.
}

Abstract

In this study, we present a homogenization model for the macroscopic rheological behavior of non-colloidal suspensions of initially spherical, viscoelastic particles in yield stress fluids, which are subjected to uniform flow conditions. The constitutive behavior of the suspending fluid is characterized by the Herschel-Bulkley (HB) model, and the particles are assumed to be neutrally buoyant solids characterized by the finite-strain Kelvin-Voigt viscoelastic behavior. We make use of the "linear comparison composite" variational technique of Ponte Castañeda (J. Mech. Phys. Solids, vol 39, 1991, pp. 45-71) to approximate the instantaneous response of the suspensions of viscoelastic particles in the HB fluid by that of a fictitious suspension consisting of the same particles distributed identically in a Newtonian fluid with a suitably-chosen viscosity. The response of the latter suspension is then estimated by the homogenization model, recently developed by Avazmohammadi and Ponte Castañeda (J. Fluid Mech., vol 763, 2015, pp. 386-432), which, when combined with appropriate evolution laws for the relevant microstructural variables, provides a complete characterization of the time-dependent response of the actual suspensions. With the objective of illustrating the key features of our model, we consider the particular case of suspensions of elastic particles in HB fluids under shear flow conditions. The results provide a broad picture of the influence of the HB fluid and

\footnotetext{
${ }^{*}$ Corresponding author, Tel: (+1) 512-232-7772, Fax: (+1) 512-471-8694.

Email addresses: rezaavaz@ices.utexas.edu (Reza Avazmohammadi), ponte@seas. upenn. edu (Pedro Ponte Castañeda)
} 
particle constitutive properties, as well as of the particle volume fraction on the effective time-dependent and steady-state behaviors of the suspensions. For the special case of non-deformable particles, our model predicts that the suspensions behave like HB fluids with modified properties, consistent with the results of Chateau et al. (J. Rheol., vol. 52, 2008, pp. 489-506).

Keywords: Yield stress fluid; Soft particle; Suspension; Shear flow

\section{Introduction}

Suspensions of micron-sized, deformable particles in a non-Newtonian fluid constitute a large class of industrial fluids including filled polymers, food pastes cosmetics and so on. The study of the rheological behavior of these suspensions is important in order to understand and tune the flow properties for a desired application. In particular, we are interested in suspensions of particles in yield stress fluids. These fluids exhibit a solid-like behavior before the stress reaches a threshold value (known as the yield stress), beyond which they behave as liquids with a non-linear stress-strain rate relation. The mechanical behavior of these fluids can be well described by the Herschel-Bulkley (HB) model. Based on this model, the yield stress fluids behave as rigid solids when subjected to shear stresses lower than the yield stress, and once the yield stress is exceeded, they flow with a nonlinear stress-strain rate relationship, either as shear-thinning, or as shear-thickening fluids. Roughly speaking, the HB model combines the classical Bingham model for simple rate-independent yield stress behavior with the power-law model for rate-dependent viscoplastic behavior, and therefore, it provides an enhanced constitutive description to capture both yield stress and rate-dependent behaviors of a broad class of non-Newtonian fluids.

The problem of predicting the rheological behavior of the suspension of rigid particles in a yield stress fluid has often been addressed in the literature by experimental and/or empirical means (see $[1,2,3,4]$, to cite only a few). On the other hand, homogenization methods developed in the context of nonlinear heterogeneous media have emerged as a promising theoretical approach to address this problem. In particular, Ponte Castañeda [5] proposed a variational principle which can be used to 
obtain bounds and estimates for the properties of nonlinear composite materials from the corresponding bounds and estimates for the effective properties of an optimally chosen "linear comparison composite" (LCC). Over the past 20 years, applications of this method have been mainly explored for viscoplastic solid composites $([6,7]$, among others). However, through the well-known analogy between the constitutive behavior of a viscoplastic solid and a yield stress fluid, some authors have applied this method to address the effective behavior of suspensions of rigid particles in yield stress fluids. Among them, Ponte Castañeda [8] considered applications of a more refined variational approach [9] for suspensions of two-dimensional (circular), rigid particles in a Bingham fluid which is a special case of the HB fluid (corresponding to $n=1$ ). Also, more recently, making use of the original variational approach, Chateau et al. [10] generated estimates for the effective viscosity of suspensions of rigid spherical particles in HB fluids. In these studies, the authors investigated the purely mechanical contribution of an isotropic distribution of rigid spherical particles to the response of yield stress fluids, and they found that the variational estimates for the effective behavior of these suspensions predict the same type of behavior as in the corresponding plain yield stress fluid with appropriately modified material constants for the fluid suspensions. Moreover, in related work, Férec et al. [11] developed a microstructure-based constitutive model for the rhelogical behavior of suspensions of rigid elongated fibers in an HB fluid, following the micromechanical framework of Gibson and Toll [12] for the case of suspensions of highly concentrated slender rigid fibers in a power-law matrix. Their constitutive model predicts a yield stress behavior for the suspension of rigid fibers, but, in general, does not follow a Herschel-Bulkley law.

The above-mentioned studies are limited to the case of non-deformable (rigid) particles. However, when the particles are deformable, it is known that their deformability and constitutive properties can strongly influence rheology of suspensions of these particles in a viscous fluid. Therefore, it is both of theoretical and practical interests to investigate how the deformability and constitutive properties of the particles suspended in a yield stress fluid affects the homogenized behavior of the 
suspension. To the best of our knowledge, the theoretical investigations of this kind has yet to be considered in the literature, although there are several studies dealing with the rheology of suspensions of deformable particles in a Newtonian fluid. In pioneering work, Roscoe [13] studied the steady-state behavior of dilute suspensions of solid viscoelastic spheres in a Newtonian fluid allowing the finite deformations of the particles. In closely related work, Goddard and Miller [14] investigated the time-dependent behavior of a viscoelastic sphere in a Newtonian fluid within the limits of small deformations of the particle. Later on, Goddard [15] studied the time-dependent rheological properties of highly concentrated suspensions of elastic particles in a Newtonian fluid, again, within the small deformation approximation. In a more recent study, making use of a polarization technique, Gao et al. [16] developed a finite-strain method to characterize the time-dependent response of dilute suspensions of neo-Hookean particles in a Newtonian fluid under Stokes flow conditions. They considered applications of their method under shear flow [17] and extensional flow [18] conditions. Most recently, building on the work of Gao et al. [16], Avazmohammadi and Ponte Castañeda [19] developed a homogenization model to estimate the time-dependent rheological behavior of non-dilute suspensions of soft viscoelastic spheres in a Newtonian fluid, again, under Stokes flow conditions. In this study, the authors formulated the homogenization problem for the suspensions in terms of the corresponding problem for a composite system whose local behavior is mathematically analogous to "thermoelastic" composites. Based on this analogy, the authors made use of the Hashin-Shtrikman-Willis (HSW) homogenization theory $[20,21]$ and provided homogenized estimates for the instantaneous response of the suspensions, together with appropriate evolution laws for the relevant microstructural variables. The distinct advantage in the exact solution of Gao et al. [16] and, similarly, in the associated estimates of Avazmohammadi and Ponte Castañeda [19], is that they account for the simultaneous deformation and rotation of particles in the macroscopic time-dependent behavior of the suspensions of the particles, when subjected to uniform flows. The results of these works in the steady state limit were successfully compared with those provided in the earlier work of Roscoe [13]. Finally, 
it is relevant to mention that, Ducloué et al. [22, 23] investigated experimentally the rheological behavior of suspensions of bubbles in yield stress fluids, and observed that these suspensions, in general, exhibit yield stress behavior, and transient viscoelastic and steady-state regimes when subjected to shear flow.

Our goal in the present study is to investigate the rheological properties of nondilute suspensions of deformable viscoelastic particles in yield stress fluids using a homogenization approach in the regime of arbitrarily large deformations. Dense suspensions of colloidal particles are typically at the origin of the yielding behavior in these fluids. However, in this study, we focus on the suspensions of non-colloidal particles in these fluids, assuming separation of length scale between the fluid microstructure and the non-colloidal particles. In other words, we assume that the size of the viscoelastic particles is much larger than the typical size of the colloidal particles so that we can neglect Brownian forces acting on the viscoelastic particles. Also, we confine our attention to the Stokes flow regime (i.e., $R e \rightarrow 0$ ), where viscous forces dominate over the inertial effects. Towards our goal, we make use of the variational approach of Ponte Castañeda [5] to replace the homogenization problem for finding the effective (instantaneous) behavior of suspensions of viscoelastic particles in yield stress fluids by a corresponding problem for a (fictitious) LCC, consisting of the same viscoelastic particles suspended in a Newtonian fluid with a suitably-chosen viscosity, denoted by $\eta^{(1)}$. The latter problem is, in fact, the very same problem addressed in [19]. Therefore, we recall pertinent estimates for the LCC from [19], which together with optimality conditions for the unknown value of $\eta^{(1)}$, can be used to obtain corresponding estimates for the instantaneous response of the suspensions of actual interest. Finally, these estimates, when combined with nonlinear equations for the evolution of the stress field in the particles, as well as for the shape and orientation of the particles, can be used to characterize the macroscopic, time-dependent rheological behavior of the suspensions in uniform flows.

The structure of the paper is as follows. Section 2 addresses the constitutive behavior of the suspending fluid and the deformable particles, as well as the types of particulate microstructures considered in this work. We consider suspensions of 
Kelvin-Voigt (KV) viscoelastic particles in yield stress fluids whose rheological behavior is characterized by the HB model. In section 3, we lay out the homogenization strategy. We first approximate the instantaneous macroscopic stress in the suspensions by that of a suitably-chosen LCC, which can in turn be given in terms of the macroscopic strain-rate tensor and the current values of the average stress inside the particle, together with the relevant microstructural variables. Next, we make use of the consistent evolution equations for these variables and the particle stress to obtain the time-dependent macroscopic response of the suspensions. Lastly, in this section, we provide explicit expressions for the particle stresses and the microstructural variables at a steady state. In section 4, we apply our model to suspensions of initially spherical, Gent hyperelastic particles (which are a special case of KV particles in the purely elastic limit) in HB fluids subjected to a shear flow, and provide representative numerical examples. Finally, some conclusions are drawn in section 6 . 


\section{Nomenclature}

D

Particle volume fraction [-]

$D_{e q}$

Strain-rate tensor $[1 / \mathrm{s}]$

$J_{m}$

Equivalent strain-rate $[1 / \mathrm{s}]$

K

Particle strain-locking parameter $[-]$

$n$

$n$

Fluid consistency constant $\left[\mathrm{Ns}^{n} / \mathrm{m}^{2}\right]$

$t$

Fluid power-law index [-]

Time $[\mathrm{s}]$

$w_{1}, w_{2}$

Particle aspect ratios [-]

$W$

Modified dissipation potential $\left[\mathrm{N} / \mathrm{m}^{2} \mathrm{~s}\right]$

W

Vorticity tensor $[1 / \mathrm{s}]$

$\dot{\gamma}$

Shear strain rate $[1 / \mathrm{s}]$

$\eta$

Viscosity constant $\left[\mathrm{Ns} / \mathrm{m}^{2}\right]$

$\theta$

Rotation angle of the particle [Rad]

$\mu$

Particle shear modulus $\left[\mathrm{N} / \mathrm{m}^{2}\right]$

$\tau_{0}$

Fluid yield stress $\left[\mathrm{N} / \mathrm{m}^{2}\right]$

$\phi$

Viscous dissipation potential $\left[\mathrm{N} / \mathrm{m}^{2} \mathrm{~s}\right]$

Special notations

$\nabla$

$(\cdot)$

Upper-convected time derivative

$(\cdot)^{(1)}$

Fluid phase variables

$(\cdot)^{(2)}$

Particle phase variables

$(\bar{\cdot})$

Macroscopic variables

$(\bar{\cdot})^{(r)}, r=1,2$

Phase average variables

$(\cdot)_{L}$

Linear comparison composite variables

\section{Suspensions of viscoelastic particles in a nonlinear viscous fluid}

In this work, we consider random suspensions of viscoelastic particles in a HerschelBulkley (HB) fluid (matrix phase). We assume that the particles and fluid phases 
have the same density, so that the particles are neutrally buoyant in the fluid. In this section, we first describe in some detail the local constitutive behavior of the fluid and particle phases, and then define pertinent microstructural variables serving to characterize the instantaneous microstructure of the suspensions. Following this section, our aim will be to deliver estimates for the macroscopic rheological response and associated microstructure evolution in these suspensions under uniform flow conditions.

We begin with a brief review of the basic kinematic relations. Under the application of mechanical loadings, the deformation gradient tensor $\mathbf{F}=\operatorname{Grad} \mathbf{x}$ serves to characterize the deformation of the material where $\mathbf{X}$ and $\mathbf{x}(\mathbf{X}, t)$ denote the position of a material point in the reference configuration and in the deformed configuration at time $t$, respectively. In the Eulerian description, the velocity gradient tensor $\mathbf{L}=\operatorname{grad} \mathbf{v}$ serves to characterize the deformation rate with $\mathbf{v}(\mathbf{x}, t)$ denoting the velocity field. We will also make use of the Eulerian strain-rate and vorticity tensors, given by $\mathbf{D}=\frac{1}{2}\left(\mathbf{L}+\mathbf{L}^{T}\right)$ and $\mathbf{W}=\frac{1}{2}\left(\mathbf{L}-\mathbf{L}^{T}\right)$, respectively.

\subsection{Constitutive behavior of the phases}

\subsubsection{Viscoelastic particles}

We assume that the suspended particles are homogeneous and made of incompressible, isotropic solids. In this work, we consider viscoelastic particles whose constitutive behavior is characterized by a finite-strain Kelvin-Voigt (KV) model. This model consists of a hyperelastic spring and a dashpot connected in parallel. For the incompressible KV material, the Cauchy stress tensor $\boldsymbol{\sigma}$ can be written as [24]

$$
\boldsymbol{\sigma}=-p_{0} \mathbf{I}+\boldsymbol{\tau}, \quad \boldsymbol{\tau}=\boldsymbol{\tau}_{e}+\boldsymbol{\tau}_{v}
$$

where $p_{0}$ is an arbitrary hydrostatic pressure due to the incompressibility constraint, I is the identity tensor, and $\boldsymbol{\tau}_{e}$ and $\boldsymbol{\tau}_{v}$ are the elastic and viscous parts of the total "extra" stress tensor $\boldsymbol{\tau}$ in the particle, respectively. In general, $\boldsymbol{\tau}$ is not deviatoric $(\operatorname{tr}(\boldsymbol{\tau}) \neq 0)$, so that the actual hydrostatic pressure $p=-\operatorname{tr}(\boldsymbol{\sigma}) / 3$ is given by $p=$ $p_{0}-\operatorname{tr} \boldsymbol{\tau} / 3$. 
The elastic stress $\boldsymbol{\tau}_{e}$ may be described in terms of a stored-energy function $\psi(\mathbf{C})$, through

$$
\boldsymbol{\tau}_{e}=2 \mathbf{F} \frac{\partial \psi(\mathbf{C})}{\partial \mathbf{C}} \mathbf{F}^{T}, \quad \operatorname{det}(\mathbf{F})=1,
$$

where $\mathbf{C}=\mathbf{F}^{T} \mathbf{F}$ is the right Cauchy-Green tensor. Avazmohammadi and Ponte Castañeda [19] presented a fairly general form of the function $\psi(\mathbf{C})$, describing generalized neoHookean behavior, to characterize the elastic part of the isotropic KV particles. In this work, we make use of a special case of this form known as the Gent constitutive model [25]. Making use of the Gent energy function (given by relation (2.6) in [19]), the corresponding extra stress tensor $\boldsymbol{\tau}_{e}$ can be expressed as

$$
\boldsymbol{\tau}_{e}=\frac{\mu J_{m}}{J_{m}-I+3} \mathbf{B}-\mu \mathbf{I}, \quad \operatorname{det}(\mathbf{F})=1,
$$

where $\mu$ is the ground state shear modulus of the elastic particle, $\mathbf{B}=\mathbf{F F}^{T}$ is the left Cauchy-Green tensor (known as the Finger tensor), and $I=\operatorname{trace}(\mathbf{B})$. The above constitutive relation has been shown to provide good agreement with experimental data for rubber-like materials [26], as it accounts for the significant stiffening in such materials at large stretches. In particular, the dimensionless parameter $J_{m}>0$ in (3), known as the strain-locking parameter, identifies limiting value for $I-3$ at which the elastic material becomes inextensible. Note that, in the limit as $J_{m} \rightarrow \infty$, the Gent model (3) reduces to the neo-Hookean model, which ignores the stiffening at large stretches.

For our purposes in this work, it is useful to derive a rate (hypo-elastic) form of the constitutive relation (3). To this end, we make use of the upper-convected (or Oldroyd) time derivative, defined as

$$
\stackrel{\nabla}{\mathbf{A}}=\dot{\mathbf{A}}-\mathbf{L A}-\mathbf{A} \mathbf{L}^{T}, \quad \operatorname{tr}(\mathbf{L})=\mathbf{0},
$$

where $\mathbf{A}$ is a symmetric second-order tensor, and $\dot{\mathbf{A}}=(\partial \mathbf{A} / \partial t)+\mathbf{v} \cdot \boldsymbol{\nabla} \mathbf{A}$ denotes the material time derivative of the tensor $\mathbf{A}$. Taking the above time derivative from both sides of relation (3), and making use of the fact that $\stackrel{\nabla}{\mathbf{B}}=\mathbf{0}$ [24], $\stackrel{\nabla}{\boldsymbol{\tau}}_{e}$ for the Gent model (3) can be expressed as

$$
{\stackrel{\nabla}{\boldsymbol{\tau}_{e}}}_{e}=2 \mu \mathbf{D}+\frac{2}{\mu J_{m}} \operatorname{tr}\left[\mathbf{D}\left(\boldsymbol{\tau}_{e}+\mu \mathbf{I}\right)\right]\left(\boldsymbol{\tau}_{e}+\mu \mathbf{I}\right) .
$$


Also, we note that, in deriving the above relation, we have expressed the tensor $\mathbf{B}$ in term of $\boldsymbol{\tau}_{e}$ by inverting the constitutive relation (3). It is worth mentioning that the constitutive relations (3) and (5) reduce to the well-known neo-Hookean expressions [24] in the limit as $J_{m} \rightarrow \infty$, namely,

$$
\boldsymbol{\tau}_{e}=\mu(\mathbf{B}-\mathbf{I}), \quad \text { and } \quad \stackrel{\nabla}{\boldsymbol{\tau}}_{e}=2 \mu \mathbf{D}
$$

Going back to the general relations (1) for the KV material, the viscous part of the extra stress can likewise be described in terms of a dissipation potential $\phi$, which is taken to be a function of the second invariant of $\mathbf{D}$, via

$$
\boldsymbol{\tau}_{v}=\frac{\partial \phi\left(D_{e q}\right)}{\partial \mathbf{D}}, \quad \operatorname{tr}(\mathbf{D})=0
$$

where $D_{e q}=\sqrt{2 \mathbf{D} \cdot \mathbf{D}}$ is the equivalent strain-rate. In this work, for simplicity, we will restrict our attention to a quadratic form of the potential, given by $\phi(\mathbf{D})=\left(\eta^{(2)} / 2\right) D_{e q}^{2}$, where $\eta^{(2)}$ is the constant viscosity of the particle material (the superscript (2) is used to denote the particle phase). Making use of this form in (7), we find the following linear constitutive relation for the viscous stress

$$
\boldsymbol{\tau}_{v}=2 \eta^{(2)} \mathbf{D}
$$

Finally, it should be noted that the set of constitutive relations for KV particles reduce to those for purely elastic particles by taking the limit as the viscosity $\eta^{(2)}$ tends to zero. In this limit - which will be of our special interest in this work - the viscous part of the stress vanishes $\left(\boldsymbol{\tau}_{v}=\mathbf{0}\right)$ and the elastic part coincides with the total stress $\left(\boldsymbol{\tau}_{e}=\boldsymbol{\tau}\right)$. Therefore, for the case of (incompressible) Gent particles, the constitutive relation for the extra stress tensor $(\boldsymbol{\tau})$ and its evolution $(\underset{\boldsymbol{\tau}}{\nabla})$ are given by (3) and (5), respectively, with $\boldsymbol{\tau}_{e}$ being replaced by $\boldsymbol{\tau}$. At the other extreme, the constitutive relations for KV particles reduce to those for purely viscous (Newtonian) particles when the shear modulus $\mu$ goes to zero. However, in this work, we will not consider the limiting case of purely viscous particles, as our main interest is focused on "solid" particles. The viscous particles would be more relevant for "fluid" inclusions (droplets) in the context of emulsions. 


\subsubsection{Nonlinear viscous fluid}

We assume that the suspending fluid is an incompressible Herschel--Bulkley fluid. These fluids do not deform or flow until the local shear stress reaches the yield stress $\tau_{0}$. Once the yield stress is exceeded, the material flows like a fluid with a non-linear stress-strain rate relationship either as a shear-thinning fluid, or a shear-thickening one. The total (Cauchy) stress tensor $\boldsymbol{\sigma}$ in the fluid is defined by expressions (1) with $\boldsymbol{\tau}_{e}=\mathbf{0}$ and $\boldsymbol{\tau}=\boldsymbol{\tau}_{v}$, because the fluid does not have elastic properties. Also, the extra stress tensor $\boldsymbol{\tau}$ is deviatoric in the fluid, so that $p_{0}=p=-\operatorname{tr}(\boldsymbol{\sigma}) / 3$ defines the actual hydrostatic pressure in the fluid. Furthermore, the constitutive relations for the HB fluid can be written in the form [27, 28]

$$
\left\{\begin{array}{l}
\mathbf{D}=\mathbf{0}, \quad \text { if } \quad \tau_{e q}<\tau_{0}, \\
\boldsymbol{\tau}=\frac{2}{D_{e q}}\left(\tau_{0}+K D_{e q}^{n}\right) \mathbf{D}, \quad \text { if } \quad \tau_{e q} \geq \tau_{0}
\end{array}\right.
$$

where $K$ is the consistency constant, $n$ is the power-law index, and $\tau_{e q}=\sqrt{\boldsymbol{\tau} \cdot \boldsymbol{\tau} / 2}$ is the equivalent shear stress. Since the material is incompressible, the constraint $\operatorname{tr}(\mathbf{D})=0$ is enforced. For the case of $\tau_{0}=0$, the constitutive model (9) represents the class of power-law fluids, where the special case of $n=1$ corresponds to an incompressible Newtonian fluid, with $K$ being the constant viscosity of the fluid. Also, for the case of $\tau_{0}>0$ and $n=1$, the model reduces to a Bingham fluid.

\subsection{Microstructure}

In this paper, we restrict our attention to suspensions consisting of initially spherical, deformable, viscoelastic particles distributed isotropically in a Herschel-Bulkley fluid matrix. Very similar to the case of suspensions of deformable particles or capsules in a Newtonian fluid (see, e.g., $[13,16,29,19]$ ), we expect that the deformable particles suspended in a non-Newtonian fluid will change their shape and orientation when subjected to a shear flow. In particular, earlier theoretical studies for dilute concentrations of neo-Hookean particles in a Newtonian fluid (e.g., [13, 16]) demonstrated that the initial spherical particles, when subjected to a shear flow, deform exactly into a series of ellipsoidal shapes - with continuously changing aspect ratios 
and principal directions - until they reach a final steady state with a fixed shape and orientation with respect to flow direction (if such a steady state exists.) This is because, in this case, the deformation field generated inside the particle is uniform. Interestingly, recent numerical simulations for the dilute suspensions of neo-Hookean particles in a non-Newtonian (viscoelastic) fluid [30, 31] revealed that, similar to the case of a suspending Newtonian fluid, the initial spherical particles deform into a shape very close to an ellipsoid until they reach a steady state. More specifically, the authors [30] did not observe more than 2-3\% deviation of the particle shape from an exact ellipsoid throughout the deformation history of the particle. For non-dilute suspensions of particles in a non-Newtonian fluid, however, we may expect that the particles attain a shape with larger deviations from an ellipsoid depending on the value of the particle concentration - these deviations would be expected due to the larger fluctuations in the deformation fields inside the particles as a consequence of the particle interactions. Moreover, we may expect that the particle interactions would affect the orientation and relative position of individual particles such that the particles in the deformed configuration would not all exhibit precisely the same exact orientation, and the two- and higher-point correlation functions for the particle centers would cease to be isotropic. Nevertheless, as discussed in [19] (see subsection 2.2 in that paper), for the purpose of providing homogenized constitutive models for the instantaneous response of the non-dilute suspensions, as well as for the evolution of their microstructure, it is reasonable to define suitable homogenized microstructural variables serving to characterize the evolution of the "average" shape and orientation of the particles, as well as of the particle distribution statistics, as functions of the deformation. Here, we adopt the microstructural model proposed in [19] to characterize microstructure of the suspensions of interest in this work. This model generalizes the earlier model proposed by Kailasam and Ponte Castañeda [7] in the context of purely viscous systems (where the matrix and particles are characterized by general nonlinear viscous behaviors) at moderate concentrations of the particles to account for elastic effects in the particles, by including the average elastic stress in the particles as an additional microstructural variable. This model assumes that 
at the present time all the initially (at zero time) spherical particles become ellipsoidal with identical shape and orientation as described by a representative (average) ellipsoid

$$
\Omega^{(2)}=\left\{\mathbf{x}:\left|(\mathbf{Z})^{-T} \mathbf{x}\right| \leq 1\right\}
$$

where $\mathbf{Z}$ is the so-called shape tensor and $\Omega^{(2)}$ denotes the volume occupied by the particle with the "average" shape and orientation in the deformed configuration. In addition, in this microstructural model, we assume that the relative position of the particles is described by two-point correlation functions having "ellipsoidal" symmetry. As discussed in more detail in [19], we further assume, for simplicity, that the shape and orientation of the particle distribution functions are identical to those of the individual particles, as described by (10). It should be emphasized, however, that in the original model of Kailasam and Ponte Castañeda [7] it was possible to account for different evolution laws for the average shape and orientation of the particles and for the shape and orientation of the angular dependence of the corresponding two-point correlation functions. This could in principle also be done in the present context, assuming that appropriate evolution laws for the twopoint correlation functions were available. Unfortunately, this is not the case at the present time, and further research on this issue will be needed to be able to develop more sophisticated models accounting for independent evolutions for the shape and orientation of the particles and their distribution.

Figure 1 depicts a snapshot of the idealized microstructure in the suspension at the present time $t$ (note that the particle are spherical at $t=0$.) The triad $\mathbf{E}_{i}, i=1,2,3$ denotes the fixed laboratory coordinates. A schematic representation of the relevant microstructural variables is also shown in this figure. The three orthonormal vectors $\mathbf{n}_{1}, \mathbf{n}_{2}$ and $\mathbf{n}_{3}=\mathbf{n}_{1} \times \mathbf{n}_{2}$ are used to characterize the principal axes of the particle. In this principal coordinate system, the shape tensor $\mathbf{Z}$ has the matrix representation $\mathbf{Z}=\operatorname{diag}\left(z_{1}, z_{2}, z_{3}\right)$ with $z_{1}, z_{2}, z_{3}$ being the three principal semi-axes of the ellipsoid. For future references, it is convenient to define two aspect ratios $w_{1}=z_{2} / z_{1}$ and $w_{2}=z_{3} / z_{1}$ which fully characterize the shape of the particle.

Consistent with the above-described microstructural model, we choose the fol- 


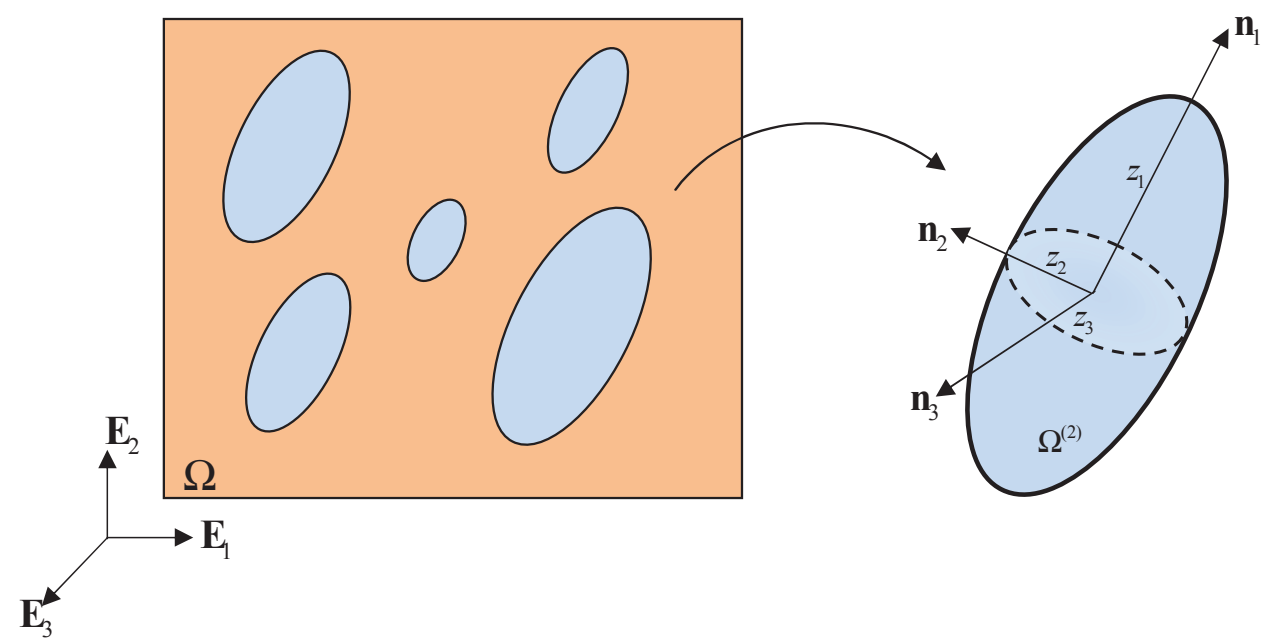

Figure 1: Graphical illustration of the microstructure in a representative volume element of the suspension occupying a volume $\Omega$ at a given time $t$. The figure shows a "representative ellipsoid" (occupying the volume $\Omega^{(2)}$ ) with aspect ratios $w_{1}=z_{2} / z_{1}$ and $w_{2}=z_{3} / z_{1}$, and with principal (orthonormal) coordinate system $\mathbf{n}_{1}, \mathbf{n}_{2}$ and $\mathbf{n}_{3}=\mathbf{n}_{1} \times \mathbf{n}_{2}$ (which rotates relative to the laboratory axes $\left\{\mathbf{E}_{i}\right\}, i=1,2,3$.)

lowing set of variables to characterize the state of the ellipsoidal microstructure:

$$
\mathscr{S}=\left\{w_{1}, w_{2}, \mathbf{n}_{1}, \mathbf{n}_{2}, \mathbf{n}_{3}=\mathbf{n}_{1} \times \mathbf{n}_{2}\right\}
$$

\section{Macroscopic response}

In this section, our aim is to determine macroscopic constitutive relations for the rheological behaviour of the suspensions described in the previous section. As discussed in subsection 2.2, the suspension, when subjected to simple flows, goes through a sequence of microstructures, approximately consisting of aligned ellipsoids that are distributed with ellipsoidal two-point statistics (with the same shape and orientation), that evolve with the deformation, starting from an initial state. Similar to our approach in [19], in this work, we break up the analysis of the macroscopic time-dependent behavior of the suspensions into two parts. In the first part, we assume that at the present instant the microstructure is given, and make use of this information to estimate the instantaneous response of the suspension by using a variational homogenization approach. In the second part, we provide consistent 
evolution equations for the relevant microstructural variables making use of the corresponding instantaneous homogenization estimates for the average deformation and stress fields in the particles. Finally, in this section, we make use of the results for the instantaneous response of the suspension and the evolution equations to provide associated estimates for the steady-state behavior of the suspension, provided that they exist. For definiteness, we use the superscripts (1) and (2) to denote variables associated with the fluid (matrix phase) and particle phase, respectively. However, for simplicity, we drop these superscripts from the variables that exist only in one phase, such as $\mu$ and $J_{m}$ in the particle phase and $K$ and $\tau_{0}$ in the fluid phase.

\subsection{Homogenization estimates for the instantaneous response}

We consider a representative volume element (RVE) of the suspension, occupying a volume $\Omega$ with boundary $\partial \Omega$. The fluid and particle phases are in turn assumed to occupy volumes $\Omega^{(1)}$ and $\Omega^{(2)}$, respectively, such that $\Omega=\Omega^{(1)}+\Omega^{(2)}$. We assume that the RVE satisfies the separation of length scales hypothesis implying that the average size of the neutrally buoyant particles is much smaller than the size of the RVE. We further assume the Stoke's condition requiring that an appropriately defined Reynolds number be small, so that inertial effects can be neglected.

Noting that the microstructure of the RVE is statistically uniform, an affine velocity boundary condition on the boundary of the RVE $(\partial \Omega)$ will produce a uniform macroscopic stress field in the RVE. Thus, the suspension is subjected to the boundary condition

$$
\mathbf{v}(\mathbf{x})=\overline{\mathbf{L}} \mathbf{x}, \quad \text { on } \partial \Omega
$$

where $\overline{\mathbf{L}}(\operatorname{tr} \overline{\mathbf{L}}=0)$ is the macroscopic, or average velocity gradient, defined by [32]

$$
\overline{\mathbf{L}}=\frac{1}{\operatorname{Vol}(\Omega)} \int_{\Omega} \mathbf{L} \mathrm{d} V
$$

It is also relevant to define the phase averages of the strain-rate and vorticity fields over phase $r(r=1,2)$ as

$$
\overline{\mathbf{D}}^{(r)}=\frac{1}{\operatorname{Vol}\left(\Omega^{(r)}\right)} \int_{\Omega^{(r)}} \mathbf{D} \mathrm{d} V, \quad \overline{\mathbf{W}}^{(r)}=\frac{1}{\operatorname{Vol}\left(\Omega^{(r)}\right)} \int_{\Omega^{(r)}} \mathbf{W} \mathrm{d} V,
$$


such that

$$
\overline{\mathbf{D}}=\frac{1}{2}\left(\overline{\mathbf{L}}+\overline{\mathbf{L}}^{T}\right)=c^{(1)} \overline{\mathbf{D}}^{(1)}+c^{(2)} \overline{\mathbf{D}}^{(2)}, \quad \overline{\mathbf{W}}=\frac{1}{2}\left(\overline{\mathbf{L}}-\overline{\mathbf{L}}^{T}\right)=c^{(1)} \overline{\mathbf{W}}^{(1)}+c^{(2)} \overline{\mathbf{W}}^{(2)},
$$

with $c^{(1)}$ and $c^{(2)}$ denoting the volume fractions of fluid and particle phases, respectively. Similarly, defining $\overline{\boldsymbol{\tau}}^{(1)}$ and $\overline{\boldsymbol{\tau}}^{(2)}$ as the averages of the extra stress tensor in the fluid and particle phases, respectively, the average or macroscopic stress can be rewritten as

$$
\overline{\boldsymbol{\sigma}}=-\bar{p}_{0} \mathbf{I}+c^{(1)} \overline{\boldsymbol{\tau}}^{(1)}+c^{(2)} \overline{\boldsymbol{\tau}}^{(2)},
$$

where $\bar{p}_{0}$ is an indeterminate hydrostatic pressure associated with the overall incompressibility constraint $(\operatorname{tr}(\overline{\mathbf{D}})=0)$ in the suspension.

Given the local constitutive relations (1), (3), and (8) for the particle phase and relation (9) for the fluid phase, and given the instantaneous microstructure (11), the effective (instantaneous) behavior of the suspension can be described by the relation between the average strain-rate tensor $\overline{\mathbf{D}}$ and the average stress tensor $\bar{\sigma}$ over the RVE $\Omega$. The problem of finding this relation can be conveniently stated by means of the minimum dissipation principles [33]. Following [19], we introduce the following pseudo-dissipation potentials

$$
W^{(r)}(\mathbf{D})=\phi^{(r)}\left(D_{e q}\right)+\boldsymbol{\tau}_{e}^{(r)} \cdot \mathbf{D}, \quad \operatorname{tr}(\mathbf{D})=0,
$$

such that the local constitutive relation of the phases can be written as

$$
\boldsymbol{\sigma}=-p_{0} \mathbf{I}+\boldsymbol{\tau}, \quad \text { where } \quad \boldsymbol{\tau}=\frac{\partial W^{(r)}}{\partial \mathbf{D}}=\frac{2}{D_{e q}} \phi^{(r)}\left(D_{e q}\right) \mathbf{D}+\boldsymbol{\tau}_{e}^{(r)} .
$$

In the above relations, $\boldsymbol{\tau}_{e}^{(r)}$ is the "elastic" stress tensor in phase $r$ which is assumed to be known at the present instant, $p_{0}$ is an indeterminate hydrostatic pressure, and $\phi^{(r)}$ denotes the (purely viscous) dissipation potentials which are only function of the equivalent strain-rate $D_{e q}$. We further emphasize that the elastic stresses $\boldsymbol{\tau}_{e}^{(r)}$ are considered to be fixed in taking the derivative with respect to $\mathbf{D}$. In particular, the local constitutive relation (18) recovers the (purely viscous) constitutive relation (9), by choosing the following properties for the fluid matrix [10]

$$
\phi^{(1)}\left(D_{e q}\right)=\tau_{0} D_{e q}+\frac{K}{n+1}\left(D_{e q}\right)^{n+1}, \quad \text { and } \quad \boldsymbol{\tau}_{e}^{(1)}=\mathbf{0} .
$$


On the other hand, relation (18) recovers the (viscoelastic) constitutive relations (3) and (8) by simply choosing

$$
\phi^{(2)}\left(D_{e q}\right)=\frac{\eta^{(2)}}{2} D_{e q}^{2} \quad \text { and } \quad \boldsymbol{\tau}_{e}^{(2)}=\boldsymbol{\tau}_{e}
$$

where $\boldsymbol{\tau}_{e}$ is characterized by the evolution equation (5), in the particle phase. Thus, it can be seen that the pseudo-dissipation potential $W^{(2)}(\mathbf{D})$, characterizing the instantaneous response of the particle, includes the effect of the elastic stress $\tau_{e}$ through the linear term in $\mathbf{D}$, where it is assumed that $\boldsymbol{\tau}_{e}$ is known at the given instant.

Next, we define the local modified dissipation potential of the suspension as

$$
W(\mathbf{x}, \mathbf{D})=\chi^{(1)}(\mathbf{x}) W^{(1)}(\mathbf{D})+\chi^{(2)}(\mathbf{x}) W^{(2)}(\mathbf{D})
$$

where the characteristic functions $\chi^{(r)}(\mathbf{x}), r=1,2$ are equal to one if the position vector $\mathbf{x}$ is in phase $r$ (i.e. $\mathbf{x} \in \Omega^{(r)}$ ) and zero otherwise. Then, making use of the principle of minimum dissipation (see, for example, [33, 19]), we can define the instantaneous macroscopic constitutive relation of the suspension, subjected to the boundary condition (12), in terms of the effective pseudo-dissipation potential $\widetilde{W}(\overline{\mathbf{D}})$ as

$$
\overline{\boldsymbol{\sigma}}=-\bar{p}_{0} \mathbf{I}+\overline{\boldsymbol{\tau}}, \quad \text { where } \quad \overline{\boldsymbol{\tau}}=\frac{\partial \widetilde{W}}{\partial \overline{\mathbf{D}}},
$$

where $\bar{p}_{0}$ is the Lagrange multiplier associated with the macroscopic incompressibility constraint and $\widetilde{W}(\overline{\mathbf{D}})$ is defined as

$$
\widetilde{W}(\overline{\mathbf{D}})=\min _{\mathbf{D} \in \mathscr{K}} \frac{1}{\operatorname{Vol}(\Omega)} \int_{\Omega} W(\mathbf{x}, \mathbf{D}) \mathrm{d} V,
$$

in which $\mathscr{K}$ denotes the set of kinematically admissible strain rates:

$\mathscr{K}=\left\{\mathbf{D} \mid\right.$ there is $\mathbf{v}$ such that $\mathbf{D}=\left(\nabla \mathbf{v}+(\nabla \mathbf{v})^{T}\right) / 2, \operatorname{div} \mathbf{v}=0$ in $\Omega$, and $\mathbf{v}=\overline{\mathbf{L}} \mathbf{x}$ on $\left.\partial \Omega\right\}$.

In the recent work by Avazmohammadi and Ponte Castañeda [19], the authors considered the homogenization problem defined by equations (22) and (23) for the instantaneous response of the viscoelastic composite system characterized by relations $(17),(18)$, and (21), when both dissipation potentials $\phi^{(1)}$ and $\phi^{(2)}$ have the 
quadratic form $\phi^{(r)}\left(D_{e q}\right)=\left(\eta^{(r)} / 2\right) D_{e q}^{2}$, with $\eta^{(r)}$ being constant-per-phase viscosity moduli. For this special case, this homogenization problem is mathematically analogous to the corresponding problem for an incompressible, linear thermoelastic composite with elastic moduli $\eta^{(r)}$ and thermal stresses $\boldsymbol{\tau}_{e}^{(r)}$ (provided that the strain-rate and velocity fields are identified with the strain and displacement fields, respectively). Therefore, in this case, the authors made use of the Hashin-ShtrikmanWillis (HSW) homogenization theory [20, 21, 34] (which has been already used for thermoelastic composites), and provided an estimate for the corresponding effective pseudo-dissipation function $\widetilde{W}(\overline{\mathbf{D}})$. However, for the problem of interest here, i.e. when $\phi^{(1)}\left(D_{e q}\right)$ is characterized by non-quadratic form (19), we propose to break up the procedure for estimating the effective function $\widetilde{W}(\overline{\mathbf{D}})$ into two parts. First, in subsection 3.1.1, we make use of the variational linear comparison method of Ponte Castañeda [5] to approximate the instantaneous behavior of the composite system, characterized by (17)-(21), by that of a linear comparison composite (LCC), consisting of the same particles suspended in a linear viscous matrix (characterized by a quadratic dissipation potential) with a suitably chosen viscosity. Second, in subsection 3.1.2, we recall pertinent estimates for the LCC from [19] to estimate the instantaneous response of the actual nonlinear composite system, characterized by $(17)-(21)$

\subsubsection{Variational estimates for the effective dissipation function}

So far, we formulated the problem of finding the effective (instantaneous) response of the suspension in terms of the effective potential $\widetilde{W}(\overline{\mathbf{D}})$ of the suspension, as described by relations $(22)$ and (23). In this work, we provide an estimate for $\widetilde{W}(\overline{\mathbf{D}})$ by making use of the variational approach in [5]. The central idea of this principle is to express the effective behavior of the nonlinear composite in terms of the effective behavior of a suitably chosen LCC which has the same microstructure (i.e. same characteristic functions $\left.\chi^{(r)}(\mathbf{x})\right)$ as the actual (nonlinear) composite material. For the class of nonlinear composites (characterized by (17)-(21)), we construct a (fictitious) LCC made of a linear viscous matrix with constant viscosity $\eta^{(1)}$ and the same particle 
phase (as characterized by properties (20)). We emphasize that the volume fraction, shape and orientation of particles in the LCC are assumed to be the same as those in the nonlinear composite at the given instant. For definiteness, the subscript $L$ has been used to denote the variables associated with the LCC.

Here, we define a LCC with local pseudo-dissipation potential

$$
W_{L}\left(\mathbf{x}, \mathbf{D} ; \eta^{(1)}\right)=\chi^{(1)}(\mathbf{x}) W_{L}^{(1)}\left(\mathbf{D} ; \eta^{(1)}\right)+\chi^{(2)}(\mathbf{x}) W_{L}^{(2)}(\mathbf{D}),
$$

where the pseudo-dissipation potentials $W_{L}^{(1)}\left(\mathbf{D} ; \eta^{(1)}\right)$ is defined as

$$
W_{L}^{(1)}\left(\mathbf{D} ; \eta^{(1)}\right)=\eta^{(1)} \mathbf{D} \cdot \mathbf{D}, \quad \operatorname{tr}(\mathbf{D})=0,
$$

with $\eta^{(1)}$ denoting the (as yet unknown) viscosity of the linear viscous matrix. Also, as already mentioned, the particle phase (phase 2) in the LCC is the same as in the nonlinear composite, so that $W_{L}^{(2)}(\mathbf{D})=W^{(2)}(\mathbf{D})$, as defined via relations (17) and (20). Correspondingly, the local constitutive relation of the phases in the LCC can be written as

$$
\boldsymbol{\sigma}_{L}=-p^{\prime} \mathbf{I}+\boldsymbol{\tau}_{L}, \quad \text { where } \quad \boldsymbol{\tau}_{L}=\frac{\partial W_{L}^{(r)}}{\partial \mathbf{D}}=2 \eta^{(r)} \mathbf{D}+\boldsymbol{\tau}_{e}^{(r)}
$$

where $\boldsymbol{\tau}_{e}^{(1)}=\mathbf{0}$ and $\boldsymbol{\tau}_{e}^{(2)}=\boldsymbol{\tau}_{e}$, as characterized by the evolution equation (5).

Following [5], the nonlinear dissipation potential $W^{(1)}(\mathbf{D})$ can then be estimated as

$$
\begin{aligned}
W^{(1)}(\mathbf{D}) & =\inf _{\eta^{(1)}>0}\left\{W_{L}^{(1)}\left(\mathbf{D} ; \eta^{(1)}\right)+V^{(1)}\left(\eta^{(1)}\right)\right\} \\
& =\inf _{\eta^{(1)}>0}\left\{\eta^{(1)} \mathbf{D} \cdot \mathbf{D}+V^{(1)}\left(\eta^{(1)}\right)\right\},
\end{aligned}
$$

where the "error" function $V^{(1)}$ is defined by

$$
\begin{aligned}
V^{(1)}\left(\eta^{(1)}\right) & =\sup _{\mathbf{D}}\left[W^{(1)}(\mathbf{D})-\eta^{(1)} \mathbf{D} \cdot \mathbf{D}\right] \\
& =\sup _{D_{e q}}\left[\phi^{(1)}\left(D_{e q}\right)-\frac{\eta^{(1)}}{2} D_{e q}^{2}\right] .
\end{aligned}
$$

In this last equation, use has been made of relation (17) and the fact that $\boldsymbol{\tau}_{e}^{(1)}=\mathbf{0}$. It is important to mention that the above expressions for $W^{(1)}(\mathbf{D})$ and $V^{(1)}\left(\eta^{(1)}\right)$ 
assume two properties for the potential $W^{(1)}(\mathbf{D})[5,32]:(i) W^{(1)}(\mathbf{D})$ has a "weakerthan-quadratic" growth, implying that $W^{(1)}-W_{L}^{(1)} \rightarrow-\infty$ as $\mathbf{D}$ tends to infinity, and (ii) $W^{(1)}(\mathbf{D})$ is square concave in $D_{e q}$, i.e., concave in the variable $D_{e q}^{2}$. Therefore, these estimates are valid only for the case of $n \leq 1$ in (19). For the case of $n>1$, corresponding expressions can be similarly defined for $W^{(1)}(\mathbf{D})$ and $V^{(1)}\left(\eta^{(1)}\right)$, as will be discussed at the end of this subsection.

Now, making use of approximation (28) together with relation (21) in definition (23) for the effective dissipation potential, interchanging the order of infima over D and $\eta^{(1)}$, and making use of a constant viscosity $\eta^{(1)}$ in the matrix phase of the LCC, we obtain (see Ponte Castañeda [37] for more details) the following estimate for the effective potential

$$
\widetilde{W}(\overline{\mathbf{D}}) \leq \inf _{\eta^{(1)}>0}\left\{\widetilde{W}_{L}\left(\overline{\mathbf{D}} ; \eta^{(1)}\right)+c^{(1)} V^{(1)}\left(\eta^{(1)}\right)\right\}
$$

where $\widetilde{W}_{L}$ denotes the effective (modified) dissipation potential of the LCC consisting of the same particle phase suspended in a fictitious Newtonian fluid with viscosity $\eta^{(1)}$, as given by

$$
\widetilde{W}_{L}\left(\overline{\mathbf{D}} ; \eta^{(1)}\right)=\min _{\mathbf{D} \in \mathscr{K}} \frac{1}{\operatorname{Vol}(\Omega)} \int_{\Omega} W_{L}\left(\mathbf{x}, \mathbf{D} ; \eta^{(1)}\right) \mathrm{d} V .
$$

The optimality conditions in (29) and (30) generate a system of algebraic nonlinear equations for the optimal values of the variables $\eta^{(1)}$ and $D_{e q}$. First, making use of expressions (19) in (29), the function $V^{(1)}$ can be re-written as

$$
V^{(1)}\left(\eta^{(1)}\right)=\sup _{D_{e q}}\left(\tau_{0} D_{e q}+\frac{K}{n+1}\left(D_{e q}\right)^{n+1}-\frac{\eta^{(1)}}{2} D_{e q}^{2}\right) .
$$

The optimality condition in the above equation, known as the secant condition, leads to the following relation

$$
\tau_{0}+K\left(\hat{D}_{e q}\right)^{n}=\eta^{(1)} \hat{D}_{e q}
$$

where $\hat{D}_{e q}$ denotes the optimal value of $D_{e q}$ in (32). In addition, making use of (32) and noting the fact that $V^{(1)}$ is stationary with respect to $\hat{D}_{e q}$, the optimality condition in (30) reduces to

$$
\frac{\partial \widetilde{W}_{L}\left(\overline{\mathbf{D}} ; \eta^{(1)}\right)}{\partial \eta^{(1)}}=\frac{1}{2} c^{(1)} \hat{D}_{e q}^{2} .
$$


In summary, the system of equations (33) and (34) can be solved for two unknowns $\eta^{(1)}$ and $\hat{D}_{e q}$. Once these two unknowns are obtained, the effective dissipation potential $\widetilde{W}(\overline{\mathbf{D}})$ can be calculated from (30). In turn, noting that estimate (30) is stationary with respect to the variable $\eta^{(1)}$, it follows from $(22)$ and (30) that the variational estimate for the macroscopic Cauchy stress in the suspension can be obtained as

$$
\overline{\boldsymbol{\sigma}}=-\bar{p}^{\prime} \mathbf{I}+\overline{\boldsymbol{\tau}}, \quad \text { where } \quad \overline{\boldsymbol{\tau}}=\frac{\partial \widetilde{W}}{\partial \overline{\mathbf{D}}}=\frac{\partial \widetilde{W}_{L}}{\partial \overline{\mathbf{D}}}=\overline{\boldsymbol{\tau}}_{L},
$$

where $\overline{\boldsymbol{\tau}}_{L}$ denotes the macroscopic extra stress tensor in the LCC, and the second equality (in the second relation) is used instead of the inequality (in (30)), in the sense of an approximation. The above results imply that the macroscopic response of the actual composite (characterized by (17)-(21)) may be approximated by that of the LCC (characterized by (25)-(27)), calculated at the optimized value of $\eta^{(1)}$. Also, it can be shown that [35] the phase averages of the stress and strain-rate fields over each phase in the actual suspension can similarly be approximated by the same quantities in the corresponding phase in the LCC. Indeed,

$$
\overline{\mathbf{D}}^{(r)}=\overline{\mathbf{D}}_{L}^{(r)}, \quad \overline{\boldsymbol{\tau}}^{(r)}=\overline{\boldsymbol{\tau}}_{L}^{(r)}, \quad r=1,2
$$

Furthermore, for later reference, we note that the second moments of the stress and strain-rate fields in each phase in the actual suspension can also be estimated in terms of the same quantities in the corresponding phase in the LCC [35]

$$
\begin{aligned}
& \frac{1}{\operatorname{Vol}\left(\Omega^{(r)}\right)} \int_{\Omega^{(r)}}(\mathbf{D} \otimes \mathbf{D}) \mathrm{d} V=\frac{1}{\operatorname{Vol}\left(\Omega^{(r)}\right)} \int_{\Omega^{(r)}}\left(\mathbf{D}_{L} \otimes \mathbf{D}_{L}\right) \mathrm{d} V, \\
& \frac{1}{\operatorname{Vol}\left(\Omega^{(r)}\right)} \int_{\Omega^{(r)}}(\boldsymbol{\tau} \otimes \boldsymbol{\tau}) \mathrm{d} V=\frac{1}{\operatorname{Vol}\left(\Omega^{(r)}\right)} \int_{\Omega^{(r)}}\left(\boldsymbol{\tau}_{L} \otimes \boldsymbol{\tau}_{L}\right) \mathrm{d} V, \quad r=1,2 .
\end{aligned}
$$

In conclusion, we see from relations (35) that the process of determining $\overline{\boldsymbol{\sigma}}$ requires the knowledge on the effective (modified) dissipation potential $\widetilde{W}_{L}$ of the LCC defined in (31). This knowledge is available from the estimates recently provided in [19] for the effective behavior of the composite characterized by relations (25)-(27). In the next subsection, we recall the estimates for the potential $\widetilde{W}_{L}(\overline{\mathbf{D}})$ and the corresponding macroscopic constitutive relation of the LCC from [19]. Finally, as 
mentioned earlier, the variational estimate (30) is valid for the case of $n \leq 1$. In this case, it can be shown that this estimate is a rigorous upper bound [5] for the effective dissipation potential $\widetilde{W}(\overline{\mathbf{D}})$, defined by (23), provided that the estimate for the potential $\widetilde{W}_{L}$ (which will be given in the next subsection) is also a rigorous upper bound for the effective dissipation potential of the LCC, defined in (31). On the other hand, for $n>1$, the appropriate variational estimate for $W^{(1)}(\mathbf{D})$ is given by

$$
W^{(1)}(\mathbf{D}) \geq \sup _{\eta^{(1)}>0}\left\{\eta^{(1)} \mathbf{D} \cdot \mathbf{D}+V^{(1)}\left(\eta^{(1)}\right)\right\}
$$

where $V^{(1)}$ is given by (29) with supremum being replaced by infimum. Following a procedure similar to the one used above to obtain the estimate (30), the corresponding estimate

$$
\widetilde{W}(\overline{\mathbf{D}}) \geq \sup _{\eta^{(1)}>0}\left\{\widetilde{W}_{L}\left(\overline{\mathbf{D}} ; \eta^{(1)}\right)+c^{(1)} V^{(1)}\left(\eta^{(1)}\right)\right\}
$$

is obtained for the case of $n>1$, where $\widetilde{W}_{L}\left(\overline{\mathbf{D}} ; \eta^{(1)}\right)$ is still given by $(31)$. In this case, the optimality conditions (33) and (34), and the macroscopic constitutive equation (35) will still hold. Finally, it is worth mentioning that the estimate (39) would provide a lower lower bound for $\widetilde{W}(\overline{\mathbf{D}})$ if the estimate for the potential $\widetilde{W}_{L}$ is also a lower bound for the effective dissipation potential of the LCC. Otherwise, the estimate (39) would still be a perfectly valid stationary estimate for $\widetilde{W}(\overline{\mathbf{D}})$.

\subsubsection{Estimates for the linear comparison composite}

In a recent paper, Avazmohammadi and Ponte Castañeda [19] developed a homogenizationbased model for the effective behavior of suspensions of initially spherical, viscoelastic particles in a Newtonian fluid (with viscosity $\eta^{(1)}$ ) under simple flows. In particular, the authors constructed their model by formulating the problem of estimating the (instantaneous) effective behavior of the suspensions in terms of finding an estimate for the effective pseudo-dissipation potential of a composite system, characterized by local relations (25)-(27). Then, to find this estimate, the authors made use of the HSW homogenization theory, which was originally developed for isotropic elastic composites by Hashin and Shtrikman [20], and extended later for generally anisotropic elastic composites by Willis $[21,34]$. More specifically, the authors made 
use of the results in [36], which is based on the HSW theory, for particulate composites consisting of aligned ellipsoidal particles that are distributed randomly with ellipsoidal two-point correlations in a matrix of a different material. As described in the previous subsection, the composite system in the work of Avazmohammadi and Ponte Castañeda [19] (characterized by local relations (25)-(27)) is, in fact, the linear comparison composite for our problem of interest in this work. Therefore, in this subsection, we recall the relevant results for the LCC from [19] for estimating the macroscopic response of the actual suspension from (35). Before proceeding, we note that we drop the subscript $L$ from the stress and strain-rate phase averages in the LCC as they are identical to the corresponding variables in the actual suspension (see relations (36).)

Here, we recall the final estimate for the effective potential $\widetilde{W}_{L}(\overline{\mathbf{D}})$ from expression (3.17) in [19], which is written as

$$
\widetilde{W}_{L}(\overline{\mathbf{D}})=\eta^{(1)} \overline{\mathbf{D}} \cdot \overline{\mathbf{D}}+c\left(\eta^{(2)}-\eta^{(1)}\right) \overline{\mathbf{D}}^{(2)} \cdot \overline{\mathbf{D}}+\frac{c}{2} \overline{\boldsymbol{\tau}}_{e}^{(2)} \cdot\left(\overline{\mathbf{D}}^{(2)}+\overline{\mathbf{D}}\right),
$$

where

$$
\overline{\mathbf{D}}^{(2)}=\left\{\mathbb{I}^{\text {Sym }}-2(1-c)\left(\eta^{(1)}-\eta^{(2)}\right) \mathbb{P}\right\}^{-1}\left\{\overline{\mathbf{D}}-(1-c) \mathbb{P} \overline{\boldsymbol{\tau}}_{e}^{(2)}\right\}
$$

is the corresponding estimate for the average strain-rate tensor $\overline{\mathbf{D}}^{(2)}$ over the particle phase, given by relation (3.18) in [19]. In these expressions, $c=c^{(2)}$ is the volume fraction of the particles, $\overline{\boldsymbol{\tau}}_{e}^{(2)}$ is the (instantaneous) average elastic stress in the particles which is assumed to be known at the given instant, $\mathbb{P}$ is a microstructural (Eshelbytype) tensor which will be defined shortly, and $\mathbb{I}^{S y m}$ denotes the identity operator in the space of (fully) symmetric fourth-order tensors (i.e., $I_{i j k l}^{S y m}=\left(\delta_{i k} \delta_{j l}+\delta_{i l} \delta_{j k}\right) / 2$ with $\delta_{i j}$ being the Kronecker delta.) It is also relevant to provide a corresponding estimate for the average vorticity tensor in the particle phase, denoted by $\overline{\mathbf{W}}^{(2)}$, as given by relation (3.19) in [19] as

$$
\overline{\mathbf{W}}^{(2)}=\overline{\mathbf{W}}+(1-c) \mathbb{R}\left[2\left(\eta^{(1)}-\eta^{(2)}\right) \overline{\mathbf{D}}^{(2)}-\overline{\boldsymbol{\tau}}_{e}^{(2)}\right]
$$

The fourth-order microstructural tensors $\mathbb{P}$ and $\mathbb{R}$ in the above relations can be 
expressed as

$$
\begin{gathered}
\mathbb{P}=\frac{1}{4 \pi|\mathbf{Z}|} \int_{|\boldsymbol{\xi}|=1} \mathbb{H}(\boldsymbol{\xi})\left|\mathbf{Z}^{T} \boldsymbol{\xi}\right|^{-3} d S, \text { and } \\
\mathbb{R}=\frac{1}{4 \pi|\mathbf{Z}|} \int_{|\boldsymbol{\xi}|=1} \mathbb{T}(\boldsymbol{\xi})\left|\mathbf{Z}^{T} \boldsymbol{\xi}\right|^{-3} d S,
\end{gathered}
$$

where the fourth-order tensor $\mathbb{H}$ and $\mathbb{T}$ are given by

$$
H_{i j k l}=\left.\left(M_{i k} \xi_{j} \xi_{l}\right)\right|_{(i j)(k l)}, \quad T_{i j k l}=\left.\left(M_{i k} \xi_{j} \xi_{l}\right)\right|_{[i j](k l)}
$$

with $\mathbf{M}=\eta^{(1)}(\mathbf{I}-\boldsymbol{\xi} \otimes \boldsymbol{\xi})$. In addition, in the above relations, the parentheses and square brackets (enclosing indices) denote symmetric and anti-symmetric parts, respectively. The components of the microstructural tensors $\mathbb{P}$ and $\mathbb{R}$ relative to the principal axes of the ellipsoidal particles are available from Appendix D in [19]. These components are written in terms of the viscosity $\eta^{(1)}$ and the current values of the aspect ratios $w_{1}$ and $w_{2}$.

As discussed in [19] (see Appendix A in that paper), consistent with the prediction of the HSW homogenization theory, the above estimates for the instantaneous response of the LCC are based on the approximation that the local polarization fields are uniform inside the particle phase. As a consequence of this approximation, the constitutive relation for the average elastic stress field in the particle phase takes the same form as in the corresponding relation for the local field (given by (3)). Therefore, similar to local relations (1), (3) and (8), the (instantaneous) average extra stress tensor in the particles in the LCC can be expressed as

$$
\begin{aligned}
& \overline{\boldsymbol{\tau}}^{(2)}=\overline{\boldsymbol{\tau}}_{e}^{(2)}+\overline{\boldsymbol{\tau}}_{v}^{(2)}, \quad \text { where } \\
& \overline{\boldsymbol{\tau}}_{e}^{(2)}=\frac{\mu J_{m}}{J_{m}-\bar{I}^{(2)}+3} \overline{\mathbf{B}}^{(2)}-\mu \mathbf{I}, \quad \text { and } \quad \overline{\boldsymbol{\tau}}_{v}^{(2)}=2 \eta^{(2)} \overline{\mathbf{D}}^{(2)},
\end{aligned}
$$

with $\overline{\boldsymbol{\tau}}_{v}^{(2)}$ denoting the viscous part of the average stress in the particles. In the above expressions, $\bar{I}^{(2)}=\operatorname{tr}\left(\overline{\mathbf{B}}^{(2)}\right), \overline{\mathbf{B}}^{(2)}=\overline{\mathbf{F}}^{(2)}\left(\overline{\mathbf{F}}^{(2)}\right)^{T}$ and $\overline{\mathbf{F}}^{(2)}$ is the average deformation gradient in the particles. It can be shown [38] that the particle phase averages $\overline{\boldsymbol{\tau}}_{e}^{(2)}, \overline{\boldsymbol{\tau}}_{v}^{(2)}$ and $\overline{\mathbf{B}}^{(2)}$ in the LCC are equal to the corresponding phase averages in the actual suspension. We also note that although the exact solution for the fields in the particles is not uniform, the uniform-field approximation is expected to lead to fairly 
accurate results provided that the concentrations are not large enough to generate strong interactions between the particles. For a detailed discussion on the limits of the validity of the LCC estimates, the interested reader is referred to Section 3 in [19].

At this point, the (instantaneous) macroscopic constitutive relation for the (actual) suspension of viscoelastic particles can be obtained by making use of estimate (40) for $\widetilde{W}_{L}(\overline{\mathbf{D}})$ in equation (35). However, Avazmohammadi and Ponte Castañeda [19] obtained a simpler (and equivalent) form of this constitutive relation (i.e., $\left.\overline{\boldsymbol{\sigma}}_{L}=-\bar{p}^{\prime} \mathbf{I}+\partial \widetilde{W}_{L} / \partial \overline{\mathbf{D}}\right)$ in terms of $\overline{\mathbf{D}}^{(2)}$ and $\overline{\boldsymbol{\tau}}_{e}^{(2)}$, which can be expressed as (see relation (3.22) in that paper)

$$
\overline{\boldsymbol{\sigma}}=-\bar{p}^{\prime} \mathbf{I}+2 \eta^{(1)} \overline{\mathbf{D}}+2 c\left(\eta^{(2)}-\eta^{(1)}\right) \overline{\mathbf{D}}^{(2)}+c \overline{\boldsymbol{\tau}}_{e}^{(2)}
$$

In summary, we see from the above constitutive relation together with expression (41) for $\overline{\mathbf{D}}^{(2)}$ that, for given macroscopic strain-rate $\overline{\mathbf{D}}$, particle volume fraction $c$, and viscosity $\eta^{(2)}$, the (instantaneous) macroscopic Cauchy stress tensor $\overline{\boldsymbol{\sigma}}$ may be determined in terms of the optimal value of $\eta^{(1)}$, the current value of the average elastic stress tensor over the particle $\overline{\boldsymbol{\tau}}_{e}^{(2)}$, and the current values of the average aspect ratio and orientation of the particles, as defined by expression (11). We obtain the optimal value of $\eta^{(1)}$ by making use of the expressions (40) in equation (34) and solving the (generally coupled) system of equations (33) and (34). This value is determined in terms of $\overline{\mathbf{D}}, c, \eta^{(2)}$, the constitutive properties of the HB fluid in the actual suspension, and the current values of the variables $\overline{\boldsymbol{\tau}}_{e}^{(2)}$ and $\mathscr{S}=\left\{w_{1}, w_{2}, \mathbf{n}_{1}, \mathbf{n}_{2}, \mathbf{n}_{3}=\mathbf{n}_{1} \times \mathbf{n}_{2}\right\}$. Therefore, the constitutive relation (48) for the instantaneous response of the actual suspension is indeed nonlinear in $\overline{\mathbf{D}}$, as expected. In the next subsection, we address the characterization of the variables $\overline{\boldsymbol{\tau}}_{e}^{(2)}$ and $\mathscr{S}$ by means of appropriate evolution equations, starting from an appropriate initial state where the particles are initially spherical and unstressed. Finally, it is instructive to note that the variational approach presented in Section 3.1.1 can be generally used to obtain bounds and estimates for the effective rheological behavior of the actual nonlinear suspensions from, not only the HSW estimate provided in this section, but any bounds and estimates 
that may be available for the effective rheological behavior of the LCC.

\subsection{Evolution equations for the microstructural variables and particle elastic stress}

So far, we generated estimates for the instantaneous response of the suspension for the current state of the microstructure and the current value of the particle elastic stress $\overline{\boldsymbol{\tau}}_{e}^{(2)}$. However, under applied flows, the microstructure in the suspension,

as well as the value of $\overline{\boldsymbol{\tau}}_{e}^{(2)}$, will generally evolve in time as the applied deformation progresses. Therefore, in order to determine the effective time-dependent behavior of suspensions from a given instantaneous state of the microstructure and the particle stress, it is essential to establish appropriate evolution laws for relevant microstructural variables and for the stress tensor $\overline{\boldsymbol{\tau}}_{e}^{(2)}$. Recalling that the microstructure as well as the stress and strain-rate phase averages in the suspension are identical to those in the LCC at any given instant, use will be made of those estimates for the actual suspensions. Therefore, the evolution of such variables may be treated in precisely the same way as in the linear comparison problem considered earlier in $[19]$.

Before proceeding with the details of the relevant evolution laws, it is important to recall from our discussion in section 2.2 that, in this study, we make use of a homogenization approach to define and estimate the microstructural variables serving to characterize the "average" shape and orientation of the particles, as opposed to being concerned with detailed shape and orientation of each individual particle. In particular, the HSW homogenization theory, used in this work, provides us with estimates for the evolution of these microstructural variables based on the approximation that the local fields are uniform inside the particle phase in the suspension. Following this approximation, and as already anticipated in section 2.2 , the initially spherical particles will deform "on average" into aligned ellipsoids with a shape and orientation that evolve with the deformation. Therefore, the set of equations used to determine the instantaneous stress and strain-rate fields inside particles will still apply at each increment of time, except that at each step the current values of the microstructural variables (including shape and orientation of the particles) and of 
the stresses in the particles will need to be used.

First, it follows from the incompressibility of the fluid and particle phases that the volume fraction of the particles will remain un-changed throughout any deformation process, i.e.,

$$
c=\text { const. }
$$

Next, the evolution for the aspect ratios of the particle, $w_{1}$ and $w_{2}$, are obtained by simple kinematic arguments (see, for example, [39]) as

$$
\dot{w}_{1}=w_{1}\left(\bar{D}_{22}^{(2)}-\bar{D}_{11}^{(2)}\right), \quad \dot{w}_{2}=w_{2}\left(\bar{D}_{33}^{(2)}-\bar{D}_{11}^{(2)}\right)
$$

where it is noted that the overdot here denotes simple time derivatives (since $w_{1}$ and $w_{2}$ depend only on time). It is also remarked in this context that the components of the tensorial variables associated with the particle phase, here and elsewhere, are referred to the principal directions of the ellipsoidal particle in their current state, as given by the triad $\left\{\mathbf{n}_{1}, \mathbf{n}_{2}, \mathbf{n}_{3}\right\}$.

Also, the evolution of the orthonormal vectors $\mathbf{n}_{1}, \mathbf{n}_{2}$ and $\mathbf{n}_{3}$, serving to characterize the orientation of particles, are determined by means of the kinematical relations

$$
\dot{\mathbf{n}}_{i}=\Omega \mathbf{n}_{i}, \quad i=1,2,3,
$$

where $\Omega$ is the (antisymmetric) spin tensor of the particle, whose components in the principal coordinate system $\left\{\mathbf{n}_{1}, \mathbf{n}_{2}, \mathbf{n}_{3}\right\}$ are determined by means of the following relations $[40,41]$

$$
\Omega_{i j}=\bar{W}_{i j}^{(2)}-\frac{\left(w_{i-1}\right)^{2}+\left(w_{j-1}\right)^{2}}{\left(w_{i-1}\right)^{2}-\left(w_{j-1}\right)^{2}} \bar{D}_{i j}^{(2)}, \quad i \neq j .
$$

In this notation, when $i$ or $j$ is equal to 1 , we define $w_{1-1}=w_{0}=1$.

Finally, an evolution equation for the particle stress $\overline{\boldsymbol{\tau}}_{e}^{(2)}$ can be obtained from relation $(47)_{1}$ by making use of the time derivative (4). In this connection, recalling that the HSW estimates for the local fields in the inclusions are uniform, so that $\overline{\mathbf{B}}^{(2)}=\overline{\mathbf{F}}^{(2)}\left(\overline{\mathbf{F}}^{(2)}\right)^{T}$, we first find that

$$
\begin{aligned}
\stackrel{\nabla}{\mathbf{B}}^{(2)} & =\dot{\overline{\mathbf{B}}}^{(2)}-\overline{\mathbf{L}}^{(2)} \overline{\mathbf{B}}^{(2)}-\overline{\mathbf{B}}^{(2)}\left(\overline{\mathbf{L}}^{(2)}\right)^{T} \\
& =\dot{\overline{\mathbf{F}}}^{(2)}\left(\overline{\mathbf{F}}^{(2)}\right)^{T}+\overline{\mathbf{F}}^{(2)}\left(\dot{\overline{\mathbf{F}}}^{(2)}\right)^{T}-\overline{\mathbf{L}}^{(2)} \overline{\mathbf{B}}^{(2)}-\overline{\mathbf{B}}^{(2)}\left(\overline{\mathbf{L}}^{(2)}\right)^{T}=\mathbf{0},
\end{aligned}
$$


where use has been made of $\overline{\mathbf{L}}^{(2)}=\dot{\overline{\mathbf{F}}}^{(2)}\left(\overline{\mathbf{F}}^{(2)}\right)^{-1}$. Making use of the above results, the evolution equation for $\overline{\boldsymbol{\tau}}^{(2)}$, defined in $(47)_{1}$, is expressed as

$$
\begin{aligned}
\stackrel{\overline{\boldsymbol{\tau}}}{e}_{e}^{(2)} & =\dot{\overline{\boldsymbol{\tau}}}_{e}^{(2)}-\overline{\mathbf{L}}^{(2)} \overline{\boldsymbol{\tau}}_{e}^{(2)}-\overline{\boldsymbol{\tau}}_{e}^{(2)}\left(\overline{\mathbf{L}}^{(2)}\right)^{T} \\
& =2 \mu \overline{\mathbf{D}}^{(2)}+\frac{2}{\mu J_{m}} \operatorname{tr}\left[\overline{\mathbf{D}}^{(2)}\left(\overline{\boldsymbol{\tau}}_{e}^{(2)}+\mu \mathbf{I}\right)\right]\left(\overline{\boldsymbol{\tau}}_{e}^{(2)}+\mu \mathbf{I}\right)
\end{aligned}
$$

In the above expressions, the material time derivative $\dot{\overline{\mathbf{B}}}^{(2)}$ and $\dot{\overline{\boldsymbol{\tau}}}_{e}^{(2)}$ are simple, time derivative, again, due to the fact that the local fields inside the particle are uniform (as already mentioned) and the convective terms hence vanish. Note that, following the same fact, evolution equation (54) takes the same form as in the corresponding equation for the local field, given by (5). For completeness, we note that, in the limit as $J_{m} \rightarrow \infty$ (corresponding to KV particles with a neo-Hookean elastic part), the above evolution equation simplifies to

$$
\underline{\nabla}_{e}^{(2)}=2 \mu \overline{\mathbf{D}}^{(2)}
$$

In summary, for a given macroscopic velocity gradient $\overline{\mathbf{L}}=\overline{\mathbf{D}}+\mathbf{\mathbf { W }}$, the macroscopic stress $\overline{\boldsymbol{\sigma}}$ is obtained from expression (48), where $\overline{\mathbf{D}}^{(2)}$ is given by expression (41). These quantities depend on two sets of values: $(i)$ the current values of the microstructural variables $\mathscr{S}$, as defined by expression (11), and determined by the evolution equations (50) and (51) from some known initial state, (ii) the current value of the average elastic stress $\overline{\boldsymbol{\tau}}_{e}^{(2)}$ in the particle, as determined by evolution equation (54), again from a known initial state. In this connection, note that the evolution equation for the particle axes (51) involves the average vorticity tensor in the particles, which is given in terms of other known variables by expression (42). In the next subsection, we will make use of the time-dependent solution, developed in this section, to construct steady-state solutions, when available, for the strain-rate, vorticity and stress fields inside particles.

\subsection{Steady-state estimates for the suspensions}

It is known from earlier work for dilute suspensions of neo-Hookean particles in a Newtonian fluid $[13,16,18]$ that the initially spherical particles, subjected 
to simple flows, can reach, under certain conditions, a steady state in which case the particles become ellipsoidal with a fixed shape and orientation, while undergoing a tank-treading motion, deforming and rotating continuously with uniform stress, strain-rate and vorticity. Recently, Avazmohammadi and Ponte Castañeda [19] demonstrated that, for suspensions of Kelvin-Voigt viscoelastic particles (with a Gent elastic part) in a Newtonian fluid, such a steady state can still be available for higher concentrations of initially spherical particles. In this study, making use of the linear comparison composite technique, we generated HSW-type estimates for the instantaneous response of the non-dilute suspensions of KV particles in a HB fluid, which also lead to the results that the initially spherical KV particles, suspended in such a fluid, can still reach a steady-state tank-treading motion, when subjected to simple flows. In this subsection, we provide steady-state (SS) estimates for the kinematical and stress tensors in the particle phase. More details on the derivation of these estimates can be found in Section 4 in [19]. For definiteness, we note that all variables in this section correspond to their associated values in a steady state. Also, in this subsection, the components of all tensorial variables are refereed to the principal directions of the ellipsoidal particles in its steady state, given by the triad $\left\{\mathbf{n}_{1}, \mathbf{n}_{2}, \mathbf{n}_{3}\right\}$.

In essence, the SS estimates can be determined by setting the terms involving time derivatives equal to zero in the evolution equation for the extra stress tensor inside the particle, as well as the evolution equations for the particle shape and orientation. The resulting expressions, together with the optimality conditions (33) and (34), will provide a set of algebraic equations to be solved for the optimal values of $\eta^{(1)}$ and $\hat{D}_{e q}$, the six components of the extra stress tensor in the particle, $\overline{\boldsymbol{\tau}}^{(2)}$, the two aspect ratios, $\omega_{1}, \omega_{2}$, and the three orientational angles defined by the particle axes, $\mathbf{n}_{1}, \mathbf{n}_{2}$, and $\mathbf{n}_{3}$.

We begin by providing SS estimates for the kinematical variables. Following [19], the evolution equations (50)-(52) together with the incompressibility constraint in the particle phase $\left(\operatorname{tr}\left(\overline{\mathbf{D}}^{(2)}\right)=0\right)$, imply that, at a steady state, the normal components 
of the strain-rate tensor (relative to the particle axes) vanish,

$$
\bar{D}_{11}^{(2)}=\bar{D}_{22}^{(2)}=\bar{D}_{33}^{(2)}=0
$$

and the three components of the vorticity strain-rate tensor in the particle phase read as

$$
\bar{W}_{12}^{(2)}=\frac{1+w_{1}^{2}}{1-w_{1}^{2}} \bar{D}_{12}^{(2)}, \quad \bar{W}_{13}^{(2)}=\frac{1+w_{2}^{2}}{1-w_{2}^{2}} \bar{D}_{13}^{(2)}, \quad \bar{W}_{23}^{(2)}=\frac{w_{1}^{2}+w_{2}^{2}}{w_{1}^{2}-w_{2}^{2}} \bar{D}_{23}^{(2)} .
$$

Also, as discussed in [19] (see Section 4 in that paper), at a steady state, the shear components of the Finger tensor $\overline{\mathbf{B}}^{(2)}$ (relative to the particle axes) are zero,

$$
\bar{B}_{12}^{(2)}=\bar{B}_{13}^{(2)}=\bar{B}_{23}^{(2)}=0
$$

and its normal components are given by

$$
\bar{B}_{11}^{(2)}=\left(w_{1} w_{2}\right)^{-2 / 3}, \quad \bar{B}_{22}^{(2)}=\left(w_{1}\right)^{4 / 3}\left(w_{2}\right)^{-2 / 3}, \quad \bar{B}_{33}^{(2)}=\left(w_{1}\right)^{-2 / 3}\left(w_{2}\right)^{4 / 3}
$$

Next, making use of $(58)$ in $(47)_{1}$, we find that, at the steady state, the shear components of the elastic stress tensor $\overline{\boldsymbol{\tau}}_{e}^{(2)}$ (relative to the particle axes) are zero. In addition, we find the three remaining (normal) components of the extra stress tensor by making use of (59) in the constitutive relation $(47)_{1}$. The final results read as

$$
\begin{aligned}
& \left(\bar{\tau}^{(2)}\right)_{e 11}=\mu d_{w}\left\{J_{m}\left(w_{2}\right)^{1 / 3}\left[1-\left(w_{1} w_{2}\right)^{2 / 3}\right]+c_{w}\right\}, \\
& \left(\bar{\tau}^{(2)}\right)_{e 22}=\mu d_{w}\left\{J_{m}\left(w_{2}\right)^{1 / 3}\left[\left(w_{1}\right)^{2}-\left(w_{1} w_{2}\right)^{2 / 3}\right]+c_{w}\right\}, \\
& \left(\bar{\tau}^{(2)}\right)_{e 33}=\mu d_{w}\left\{J_{m}\left(w_{2}\right)^{1 / 3}\left[\left(w_{2}\right)^{2}-\left(w_{1} w_{2}\right)^{2 / 3}\right]+c_{w}\right\},
\end{aligned}
$$

where $c_{w}=\left(w_{2}\right)^{1 / 3}\left[\left(w_{1}\right)^{2}+\left(w_{2}\right)^{2}+1\right]-3\left(w_{1}\right)^{2 / 3} w_{2}$, and $d_{w}=\left[J_{m}\left(w_{1}\right)^{2 / 3} w_{2}-c_{w}\right]^{-1}$.

Now, making use of the fact that the shear components of the stress tensor $\overline{\boldsymbol{\tau}}_{e}^{(2)}$ are zero in (41) and (42), the non-zero components of the particle strain-rate $\overline{\mathbf{D}}^{(2)}$ and vorticity $\overline{\mathbf{W}}^{(2)}$ at the steady state can be written as

$$
\left.\bar{D}_{i j}^{(2)}=\frac{\bar{D}_{i j}}{1-\alpha \mathbb{P}_{i j i j}}, \quad \bar{W}_{i j}^{(2)}=\bar{W}_{i j}+\frac{\alpha \mathbb{R}_{i j i j} \bar{D}_{i j}}{1-\alpha \mathbb{P}_{i j i j}}, \quad i, j=1,2,3 \text { (no sum, } i \neq j\right),
$$

where $\alpha=4(1-c)\left(\eta^{(1)}-\eta^{(2)}\right)$ and all components are given relative to the particle axes. 
Next, relations (56), together with the constitutive equation $(47)_{2}$, imply that, at the steady state, the normal components of the viscous part of extra stress tensor (relative to the particle axes) in the KV particles must all vanish. Moreover, substituting the shear components of $\overline{\mathbf{D}}^{(2)}$ from $(61)_{1}$ into $(47)_{2}$, we find the (remaining) shear components of $\overline{\boldsymbol{\tau}}_{v}^{(2)}$, as follows

$$
\left(\bar{\tau}_{v}^{(2)}\right)_{i j}=\frac{2 \eta^{(2)} \bar{D}_{i j}}{1-4(1-c)\left(\eta^{(1)}-\eta^{(2)}\right) \mathbb{P}_{i j i j}}, \quad i, j=1,2,3 \quad(i \neq j) .
$$

Finally, the components of the total extra stress tensor in the KV particles at the steady state can be obtained in terms of the above estimates for the viscous and elastic extra stresses in the particles by means of relation (46).

At this stage, the optimal values of $\eta^{(1)}$ and $\hat{D}_{e q}$, all non-zero components of the particle stress $\overline{\boldsymbol{\tau}}^{(2)}$, strain-rate $\overline{\mathbf{D}}^{(2)}$ and vorticity $\overline{\mathbf{W}}^{(2)}$ are written in terms of SS values of the aspect ratios, $w_{1}$ and $w_{2}$, and the three orientational angle defined by the particles axes, $\mathbf{n}_{1}, \mathbf{n}_{2}$, and $\mathbf{n}_{3}$. We obtain a system of algebraic equations for the unknowns $w_{1}, w_{2}, \mathbf{n}_{1}, \mathbf{n}_{2}, \mathbf{n}_{3}$ by making use of (60) into (41), together with the three equations obtained by substituting relations (61) into (57), and expression (56).

Finally, we remark that the SS solutions for suspensions of KV particles reduce to those for suspensions of elastic particles by setting $\left(\bar{\tau}_{e}^{(2)}\right)_{i j}=\bar{\tau}_{i j}^{(2)}$ and $\left(\bar{\tau}_{v}^{(2)}\right)_{i j}=0$ $(i, j=1,2,3)$ in relations $(60)$ and $(46)$.

\section{Application: Shear flow}

To illustrate the main features of the constitutive model developed in Section 3, in this section, we apply the model to suspensions of initially spherical, elastic particles in a Herschel-Bulkley (HB) fluid subjected to a uniform shear flow for representative constitutive properties of the fluid and particles as well as volume fraction of particles. It should be noted that different initial shapes and viscous effects for the particles, as well as other types of flows (e.g., extensional, oscillatory, relaxation), could also be considered in the context of the general model developed in the previous sections. However, due to limitations of space, such more general particle and flow types will not be considered in this paper and will be left for future investigations. Thus, here 
we consider the problem of suspensions in a simple shear flow, characterized by

$$
\overline{\mathbf{L}}=\dot{\gamma} \mathbf{E}_{1} \otimes \mathbf{E}_{2}, \quad \overline{\mathbf{D}}=\frac{1}{2} \dot{\gamma}\left(\mathbf{E}_{1} \otimes \mathbf{E}_{2}+\mathbf{E}_{2} \otimes \mathbf{E}_{1}\right), \quad \overline{\mathbf{W}}=\frac{1}{2} \dot{\gamma}\left(\mathbf{E}_{1} \otimes \mathbf{E}_{2}-\mathbf{E}_{2} \otimes \mathbf{E}_{1}\right),
$$

where the $\left\{\mathbf{E}_{i}\right\}$ refer to the fixed laboratory coordinates, and $\dot{\gamma} \geq 0$ is the shear strain rate. For convenience, we make use of the dimensionless parameters

$$
G=\frac{K \dot{\gamma}^{n}}{\mu}, \quad \text { and } \quad H=\frac{\tau_{0}}{K \dot{\gamma}^{n}}
$$

which serve to characterize the ratio of viscous forces in the fluid to the elastic forces in the particles, and the ratio of the forces (exclusively) induced by the yield stress to the viscous forces induced by the application of the strain rate in the fluid, respectively. Also, for future reference, it is useful to introduce a (dependent) nondimensional parameter, defined by

$$
H_{G}=H G=\frac{\tau_{0}}{\mu}
$$

It is relevant to mention that the special case of $G=0$ corresponds to suspensions of (spherical) rigid particles in a HB fluid. Also, the special cases of $H=0$ and $n=1$ correspond to the suspensions of elastic particles in a power-law and Bingham fluids, respectively. All representative examples in this section are provided for the case of (purely) elastic Gent particles (i.e., $\eta^{(2)}=0$ ) with a fixed strain-locking parameter $\left(J_{m}=50\right)$.

The initially spherical particles, when subjected to shear flow (63), deform (on average) into ellipsoids of general ellipsoidal shape characterized by the two aspect ratios $w_{1}$ and $w_{2}$, which, as defined in figure 1 , correspond to the aspect ratios of the particle in the plane of the flow $\left(\mathbf{E}_{1}-\mathbf{E}_{2}\right.$ plane $)$ and in the plane perpendicular to the short in-plane axis of the particles $\left(\mathbf{E}_{1}-\mathbf{E}_{3}\right.$ plane), respectively. The deformed particles also rotate (on average) remaining in the $\mathbf{E}_{1}-\mathbf{E}_{2}$ plane in such a way that their current orientation may be described in terms of a single angle $\theta$ (measured positive in the counterclockwise direction from the $\mathbf{E}_{1}$ direction). In other words, as schematically shown in figure 1 , the principal axis $\mathbf{n}_{3}$ of the particles remains aligned with the axis $\mathbf{E}_{3}$ and the axes $\mathbf{n}_{1}$ and $\mathbf{n}_{2}$, while rotating about the axis 
$\mathbf{E}_{3}$, remain in the fixed plane $\mathbf{E}_{1}-\mathbf{E}_{2}$, throughout the deformation of the particles. Then, the evolution equations (50) to (54) can be shown to specialize to equations (A.1) in Appendix A. These equations are complemented with two more equations (33) and (34) for the unknown variables $\eta^{(1)}$ and $\hat{D}_{e q}$ associated with the LCC. The resulting system of equations, together with relations (41) and (42), can be integrated numerically for the time-dependent solution. The components of the shape tensors $\mathbb{P}$ and $\mathbb{R}$, required for this integration, are not included here for brevity, but they are available from Appendix D in [19]. Finally, given the current values of the particle stress $\overline{\boldsymbol{\tau}}^{(2)}$, aspect ratios $w_{1}$ and $w_{2}$ and orientation $\theta$ of the particles, the macroscopic stress $\overline{\boldsymbol{\sigma}}$ can be computed by means of expression (48), together with (41). The above-mentioned equations were integrated using an explicit time discretization scheme, and the typical integration time was in the order of a few minutes in a laptop.

For the specific purpose of discussing the macroscopic response of the suspensions in shear flow, we present the results for the shear component of the macroscopic Cauchy stress tensor, $\bar{\sigma}_{12}$, as well as the first and second normal stress differences of the suspensions, defined by

$$
\Pi_{1}=\bar{\sigma}_{11}-\bar{\sigma}_{22}, \quad \Pi_{2}=\bar{\sigma}_{22}-\bar{\sigma}_{33}
$$

where the components are relative to the fixed laboratory axes $\mathbf{E}_{i}$.

To obtain the initial response of the suspension, we make use of the (initial) condition $\left.\overline{\boldsymbol{\tau}}^{(2)}\right|_{t=0^{+}}=\mathbf{0}$. (We use the notation $t=0^{+}$to denote the very first instant at which the suspension flows in an average sense under the applied flow conditions.) This condition for the particle stress is due to the fact that, at $t=0^{+}$, the elastic particles have not had the time to respond to the applied flow, and therefore, at this instant, they behave like spherical, incompressible voids. Hence, making use of the Hashin-Shtrikman (HS) upper bound for the effective potential $\widetilde{W}_{L}$ of the LCC (consisting of a viscous matrix with viscosity $\eta^{(1)}$ and random distribution of incompressible, spherical voids) in equations (33) and (34), the unknown viscosity 
$\eta^{(1)}$ in the LCC is obtained as

$$
\left.\eta^{(1)}\right|_{t=0^{+}}=\sqrt{\frac{3+2 c}{3}} \frac{\tau_{0}}{\bar{D}_{e q}}+\left(\sqrt{\frac{3}{3+2 c}}\right)^{n-1} K \bar{D}_{e q}^{n-1} .
$$

where $\bar{D}_{e q}=\sqrt{2 \overline{\mathbf{D}} \cdot \overline{\mathbf{D}}}$ denotes the macroscopic equivalent strain-rate. We note that the above relation is valid for a general uniform macroscopic flow $\overline{\mathbf{D}}$. Similarly, making use of the corresponding $\widetilde{W}_{L}$ in (35), together with the above relation, we find the initial macroscopic Cauchy stress tensor as

$$
\begin{aligned}
& \left.\overline{\boldsymbol{\sigma}}\right|_{t=0^{+}}=-\bar{p}^{\prime} \mathbf{I}+\left.\overline{\boldsymbol{\tau}}\right|_{t=0^{+}}, \quad \text { where } \\
& \left.\overline{\boldsymbol{\tau}}\right|_{t=0^{+}}=\left.\frac{6(1-c)}{3+2 c} \eta^{(1)}\right|_{t=0^{+}} \overline{\mathbf{D}}=\frac{2(1-c)}{\bar{D}_{e q}}\left[c^{I} \tau_{0}+\left(c^{I}\right)^{n+1} K \bar{D}_{e q}^{n}\right] \overline{\mathbf{D}}
\end{aligned}
$$

with $c^{I}=\sqrt{3 /(3+2 c)}$. By comparing the above relation with the corresponding relation for the extra stress in a plain HB fluid (see relation $(9)_{2}$ ), it follows that, according to the variational method prediction, suspensions of elastic particles in a HB fluid with yield stress $\tau_{0}$ and consistency constant $K$ initially behave like a HB fluid with the same power index $n$ as that of the suspending fluid, but with effective yield stress $\tilde{\tau}_{0}^{I}$ and consistency constant $\tilde{K}^{I}$, given by

$$
\tilde{\tau}_{0}^{I}=(1-c) c^{I} \tau_{0}, \quad \tilde{K}^{I}=(1-c)\left(c^{I}\right)^{n+1} K .
$$

For the case of shear flow conditions (63), it follows from the general result (66) that the initial shear stress component in the suspension is given by

$$
\left.\bar{\sigma}_{12}\right|_{t=0^{+}}=\tilde{\tau}_{0}^{I}+\tilde{K}^{I} \dot{\gamma}^{n}
$$

Next, it is worthwhile to provide analytical expressions for the response of the suspension at a large enough time, specifically when the suspension reaches a steady state. Under the shear flow conditions (63), making use of (60) and $(61)_{1}$ in relation (48), the steady-state value of the macroscopic stress component $\bar{\sigma}_{12}$ is obtained as

$$
\begin{aligned}
\left.\bar{\sigma}_{12}\right|_{S S} & =\eta^{(1)} \dot{\gamma}\left(1-\frac{c \cos (2 \theta)}{1-4(1-c) \eta^{(1)} \mathbb{P}_{1212}}\right) \\
& +c \mu \sin (\theta) \cos (\theta) d_{w}\left[J_{m}\left(w_{2}\right)^{1 / 3}\left(1-w_{1}^{2}\right)\right] .
\end{aligned}
$$


where all the quantities $w_{1}, w_{2}, \theta$, and $\eta^{(1)}$ are evaluated at the steady state, and the component $\mathbb{P}_{1212}$ (relative to the particle axes) is obtained in terms of the corresponding values of $w_{1}, w_{2}$, and $\eta^{(1)}$ (see Appendix D in [19].)

Before proceeding with the detailed examples, it is also useful to consider the response of the suspensions in the limiting case of $G \rightarrow 0$ (under a constant strainrate tensor $\overline{\mathbf{D}}$ ) which corresponds to the suspension of rigid spherical particles. In this limit, the microstructure of the suspension does not evolve because the rigid particles do not change shape and the particles distribution remain spherical based on our earlier assumption in Section 2.2 that the shape and orientation of the particles distribution are identical to those of the individual particles. Therefore, since there is no microstructure evolution for the case of $G \rightarrow 0$, the steady-state response of the suspension is the same as the initial response of the suspension. In this case, making use of the HS lower bound for the effective potential $\widetilde{W}_{L}$ of the LCC (consisting of a viscous matrix with viscosity $\eta^{(1)}$ and random distribution of rigid spherical particles) in equations (33) and (34), the unknown viscosity $\eta^{(1)}$ in the LCC is obtained as

$$
\left.\eta^{(1)}\right|_{G \rightarrow 0}=(1-c) \sqrt{\frac{2}{2+3 c}} \frac{\tau_{0}}{\bar{D}_{e q}}+\left(\frac{1}{1-c} \sqrt{\frac{2+3 c}{2}}\right)^{n-1} K \bar{D}_{e q}^{n-1} .
$$

Similarly, making use of the corresponding $\widetilde{W}_{L}$ in (35) together with (70), we find the initial macroscopic Cauchy stress tensor as

$$
\begin{aligned}
& \left.\overline{\boldsymbol{\sigma}}\right|_{G \rightarrow 0}=-\bar{p}^{\prime} \mathbf{I}+\left.\overline{\boldsymbol{\tau}}\right|_{G \rightarrow 0}, \quad \text { where } \\
& \left.\overline{\boldsymbol{\tau}}\right|_{G \rightarrow 0}=\left.\frac{2+3 c}{(1-c)} \eta^{(1)}\right|_{G \rightarrow 0} \overline{\mathbf{D}}=\frac{2}{\bar{D}_{e q}} c^{R}\left\{\tau_{0}+\left[c^{R} /(1-c)\right]^{n} K \bar{D}_{e q}^{n}\right\} \overline{\mathbf{D}}
\end{aligned}
$$

where $c^{R}=\sqrt{(2+3 c) / 2}$. Again, it follows from the above form for the macroscopic stress that, according to the variational method prediction, the suspension of the rigid particles in a Herschel-Bulkley fluid will exhibit a HB behavior with the same power index $n$ as that of the suspending fluid, but with effective yield stress $\tilde{\tau}_{0}^{R}$ and consistency constant $\tilde{K}^{R}$, given by

$$
\tilde{\tau}_{0}^{R}=c^{R} \tau_{0}, \quad \tilde{K}^{R}=(1-c)\left[c^{R} /(1-c)\right]^{n+1} K .
$$


It is worth mentioning that this prediction of the variational method is in agreement with earlier results by Chateau et al. [10] for suspensions of rigid spherical particles in a HB fluid, as well as with the variational estimates reported by Ponte Castañeda [8] for suspensions of idealized 2-D, rigid circular particles in a Bingham fluid. For the case of shear flow conditions (63), it follows from the general results (71) that the shear stress component in the suspension when $G \rightarrow 0$ is written as

$$
\left.\bar{\sigma}_{12}\right|_{G \rightarrow 0}=\tilde{\tau}_{0}^{R}+\tilde{K}^{R} \dot{\gamma}^{n}
$$

It is also important to note that, when $n \leq 1$, it can be shown that the estimates (73) serves as a rigorous lower bound on the effective stress component $\bar{\sigma}_{12}$ for the case of suspensions of rigid spherical particles in a HB fluid.

The results provided in this section are organized as follows. In Sections 4.1 and 4.2, we present the results for the suspensions of Gent particles in Bingham fluids $(n=1)$ and power-law fluids $(H=0)$, respectively. Next, in Section 4.3, we will present representative results for the suspensions of Gent particles in a general HB fluid. Also, in all three subsections, results are first provided for time-dependent responses of the corresponding suspensions, followed by results for steady-state (SS) behaviors of these suspension. For both groups of results, figures present results for the average particle shape (characterized by $w_{1}$ and $w_{2}$ ) and orientation (characterized by $\theta$ ), certain components of the average extra stress tensor in the fluid phase $\left(\overline{\boldsymbol{\tau}}^{(1)}\right)$ and in the particle phase $\left(\overline{\boldsymbol{\tau}}^{(2)}\right)$, as well as the macroscopic Cauchy stress $\bar{\sigma}_{12}$ and stress differences $\Pi_{1}$ and $\Pi_{2}$, as defined in (64). We emphasize that the components of the macroscopic Cauchy stress and the average stress tensor in the fluid are shown relative to the fixed laboratory axes $\mathbf{E}_{i}$, while the components of the average stress tensor in the particle are shown relative to the instantaneous principal axes of the particle. Finally, we note that all stress components are appropriately normalized in the plots.

\subsection{Bingham fluids}

In this subsection, we present results for the case of suspensions of elastic (Gent) particles in a Bingham fluid (i.e., $n=1$ ). Figure 2 presents variational estimates for 


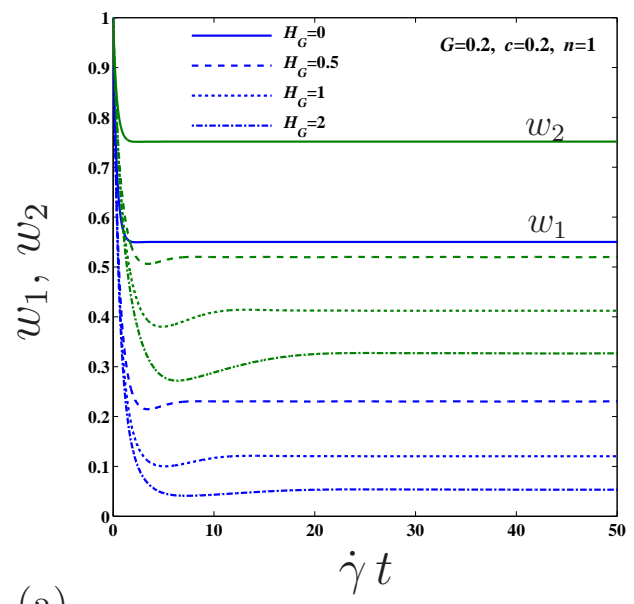

(a)

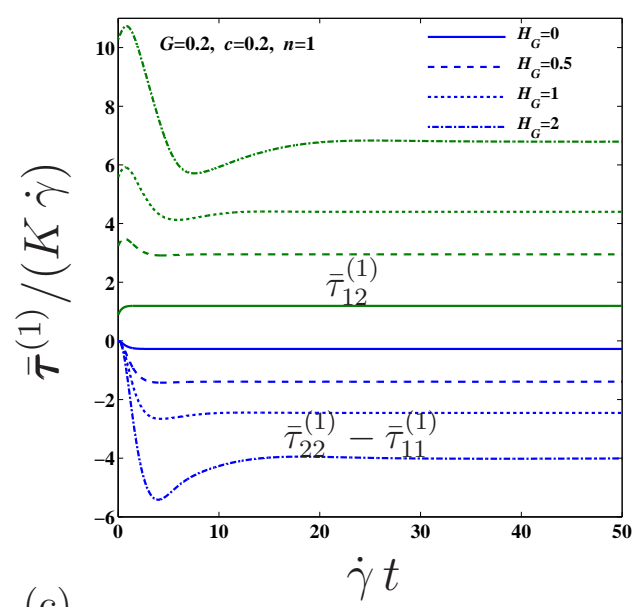

(c)

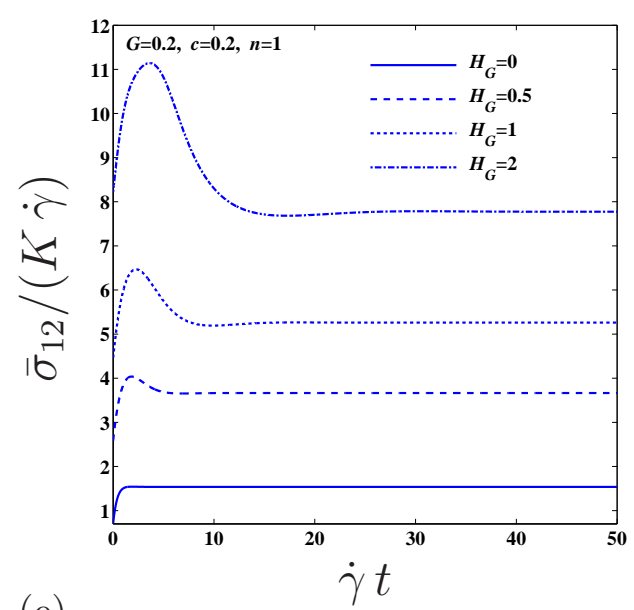

(e)

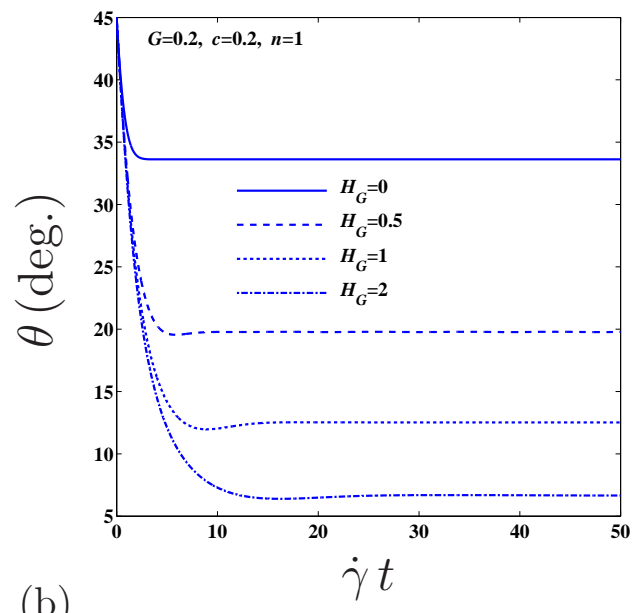

(b)

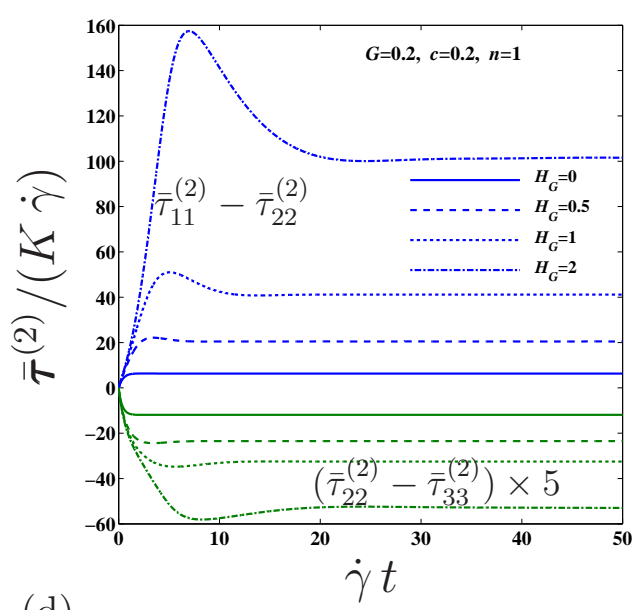

(d)

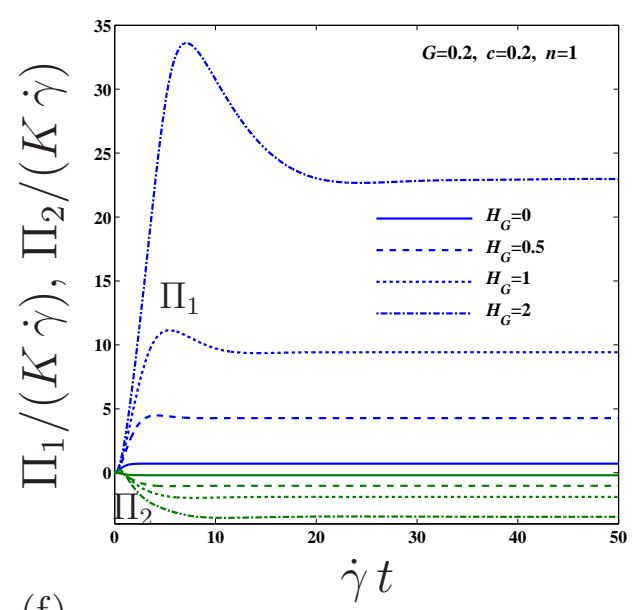

(f)

Figure 2: Variational estimates for the time-dependent response of suspensions of initially spherical Gent particles with $G=0.2$ in a Bingham fluid $(n=1)$ for various values of $H_{G}$ under shear flow conditions. The components of $\overline{\boldsymbol{\tau}}^{(1)}$ and $\overline{\boldsymbol{\tau}}^{(2)}$ are given relative to the fixed coordinate system $\mathbf{E}_{i}$ and the principal axes of the particles, respectively. 
the time-dependent behavior of suspensions of purely elastic (Gent) particles with $G=0.2$ in a Bingham fluid in simple shear. Results are shown for a fixed volume fraction $(c=0.2)$ and various values of the non-dimensional parameter $H_{G}=\tau_{0} / \mu$. It is useful to note that the results are calculated at a constant strain rate $\dot{\gamma}$ and shear modulus $\mu$, so that higher values of $H_{G}$ correspond to Bingham fluids with a higher yield stress $\tau_{0}$. In particular, figures 2 (a) and (b) show results for the evolution of the particle shape and orientation, respectively, while figures 2 (c), (d), (e), and (f) show results for the time-dependent behavior of the average stress in the fluid phase, the average stress in the particle phase, the macroscopic shear stress, and the macroscopic stress differences, respectively. A general observation from the plots in figure 2 is that all the variables exhibit a transient behavior which leads into a steady-state regime. In particular, the evolutions of $w_{1}, w_{2}$ and $\theta$ in figures 2 (a) and (b) suggest that the particles suspended in a Bingham fluid with a higher yield stress become more elongated and their major axis becomes more aligned with the shear direction, before they reach a steady state. In fact, as we observe from figure 2(c), this is because a higher yield stress of the Bingham fluid results in a larger initial stress in the fluid matrix which, in turn, induces a higher level of stress in the particles, as observed from figure 2(d). As a consequence, we observe from figures $2(\mathrm{f})$ and (e) that the macroscopic shear stress and normal stress differences exhibit a progressive increase (in magnitude) as the yield stress of the suspending fluid increases. We further observe from figures 2 (e) and (f) that the time-dependent behavior of the macroscopic shear stress and the normal stress differences exhibit an "overshoot" (before approaching the steady state), which becomes more pronounced with increasing values of $H_{G}$. We observe a similar trend in the behavior of the average stress in the particle phase from figure 2 (c), as well as in the evolution of the out-of-plane aspect ratio $\left(w_{2}\right)$ from figure $2(\mathrm{a})$. These observations suggest that the particles appear to be initially softer for Bingham fluids with a higher yield stress, but they tend to shrink back in the plane perpendicular to the short in-plane axis of the particles before reaching a steady state, which causes a drop in the average stress in the particle phase and in the macroscopic stresses. Finally, it is worth noting that 
the initial values of the (normalized) macroscopic shear stress in figure 2(e) define the initial yield stress of the suspension which is explicitly given in terms of $\tau_{0}$ and $c$ by relation (67). On the other hand, the steady-state values of the macroscopic shear stress are influenced by the evolution of the microstructure, and they can be determined from relation (69) in terms of the $\mathrm{SS}$ values of $w_{1}, w_{2}$ and $\theta$, as well as the SS value of the viscosity $\eta^{(1)}$.

Next, we investigate the steady-state response of the suspensions of Gent particles in a Bingham fluid in simple shear. Figure 3 shows variational estimates for the steady-state behavior of the particle, as well as for the associated stress averages in the phases and macroscopic stresses, as a function of $G$. The results are given for a fixed volume fraction $(c=0.2)$ and several values of $H$. We note that higher values of $H$ and $G$ correspond to higher yield stresses of the Bingham fluid and softer particles, respectively. We observe from figures $3(\mathrm{a})$ and (b) that, as $G$ increases, the Gent particles reach larger elongations, as well as closer alignments with the shear direction at the steady state. This behavior of the particles is seen to be strongly affected by the value of the yield stress of the suspending Bingham fluid. Generally, the rate of change in SS values of the aspect ratios and orientation of the particles with increasing $G$ is higher for larger values of $H$. This is, once again, due to the fact that a higher yield stress $\tau_{0}$ of the Bingham fluid leads to larger initial stresses in the fluid matrix and particle, as observed from figures 3(c) and (d), and these stresses have a stronger effect on the SS shape and orientation of the particles when $G$ is higher, corresponding to softer particles. Next, figures 3(e) and (f) show the corresponding estimates for the macroscopic shear and normal stress differences, respectively. The variable $\bar{\sigma}_{12} /(K \dot{\gamma})$ in figure $3(\mathrm{e})$ defines the dimensionless (apparent) effective viscosity of the suspension, and its decrease with $G$ is more pronounced for higher values of $H$. Moreover, when $G \rightarrow 0$, which corresponds to suspensions of rigid spherical particles, the values of the (normalized) macroscopic shear stress are in agreement with the values calculated from (73) for $c=0.2$ and corresponding values of $H$.

In figure 4, we investigate the influence of the particle volume fractions on the 


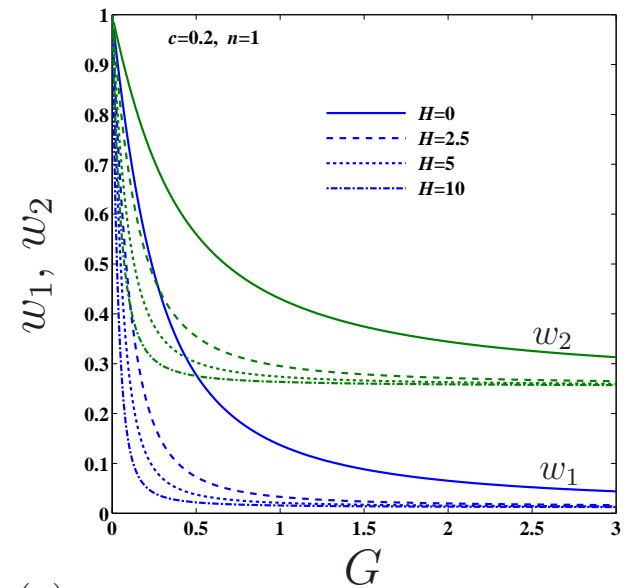

(a)

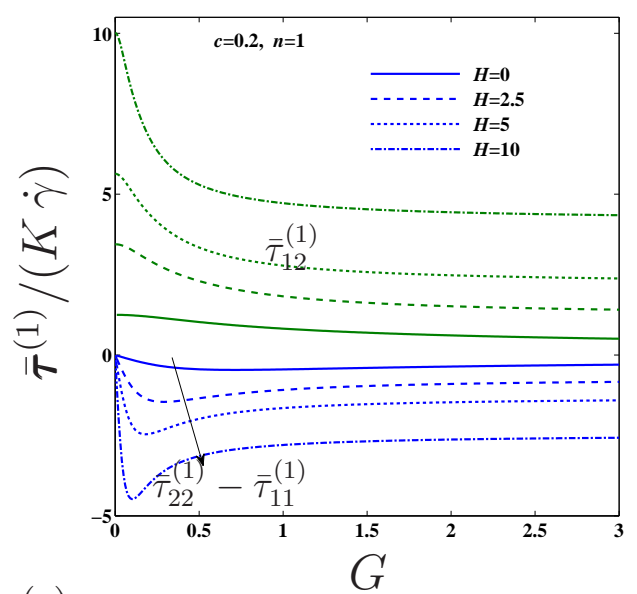

(c)

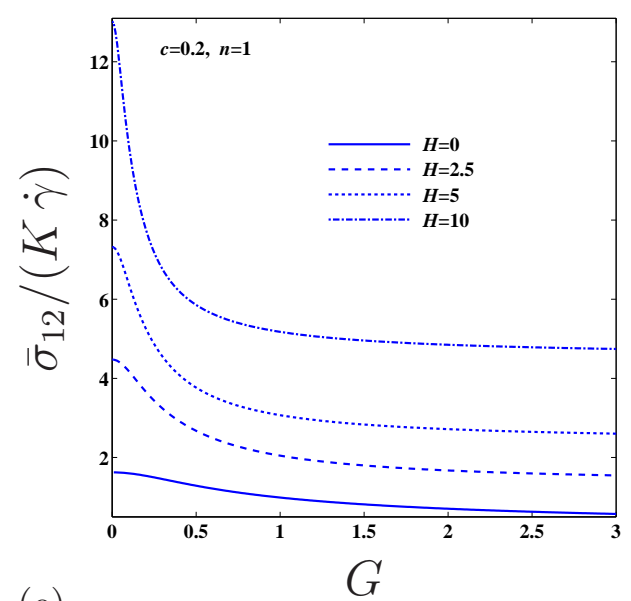

(e)

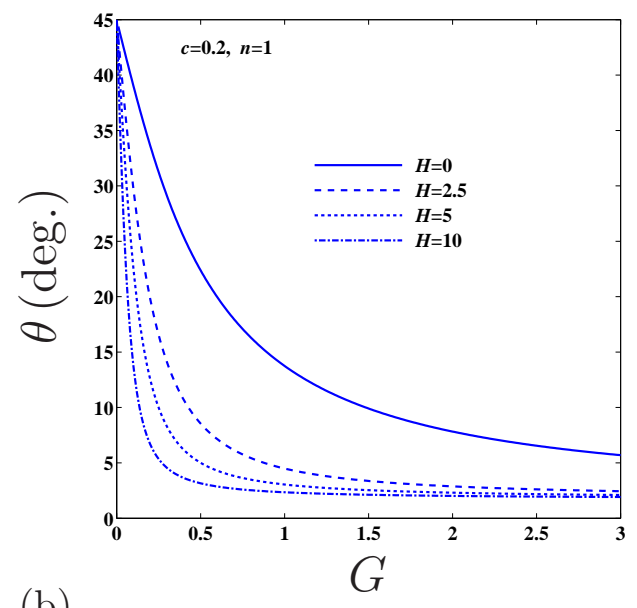

(b)

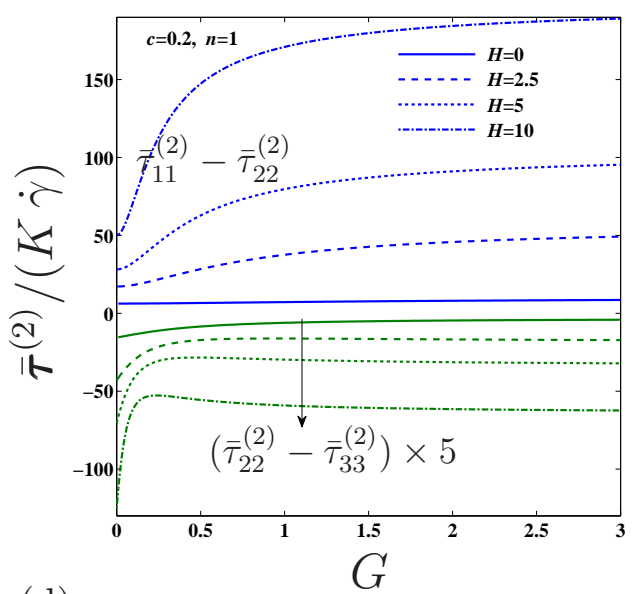

(d)

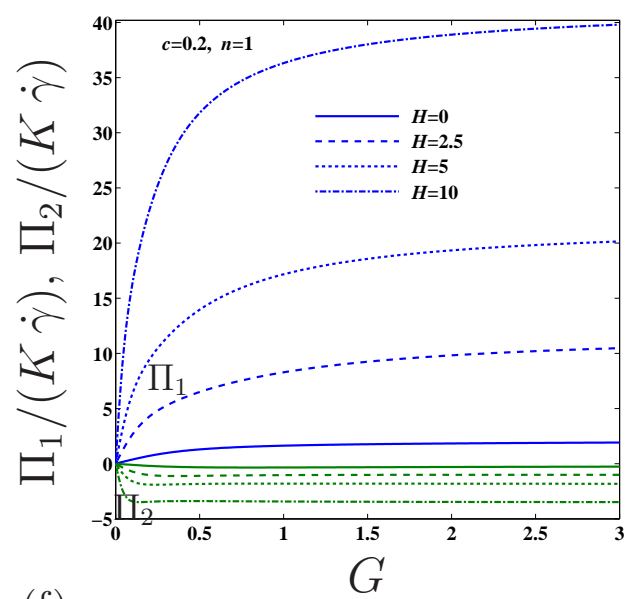

(f)

Figure 3: Variational estimates for the steady-state response of suspensions of initially spherical Gent particles with $G=0.2$ in a Bingham fluid $(n=1)$ for various values of $H$ under shear flow conditions. The components of $\overline{\boldsymbol{\tau}}^{(1)}$ and $\overline{\boldsymbol{\tau}}^{(2)}$ are given relative to the fixed coordinate system $\mathbf{E}_{i}$ and the principal axes of the particles, respectively. 


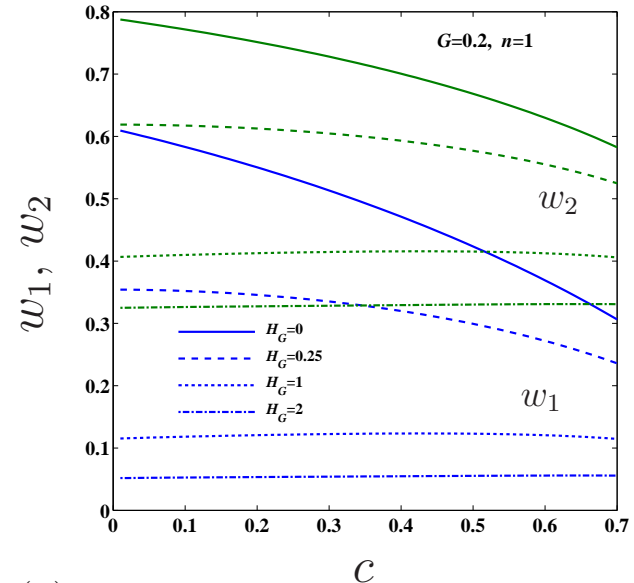

(a)

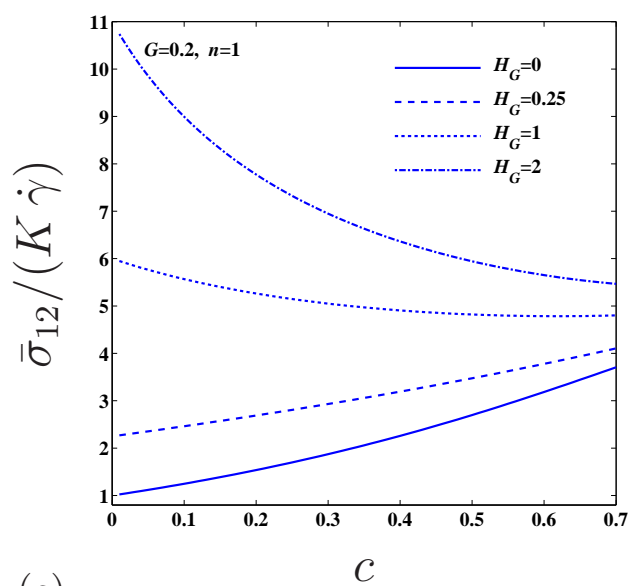

(c)

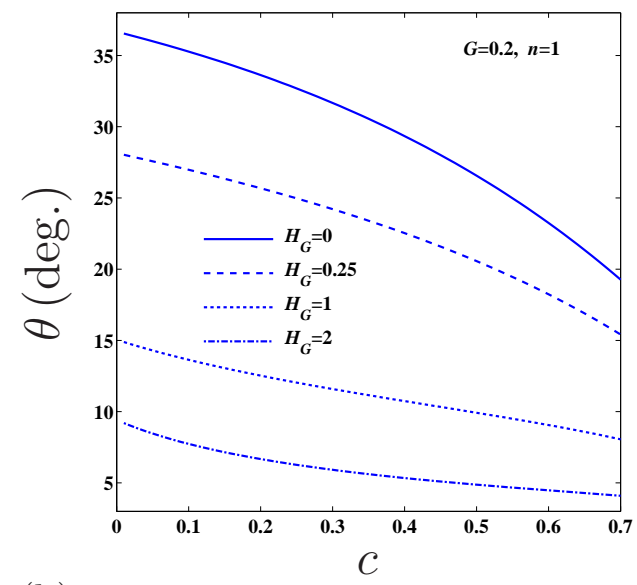

(b)

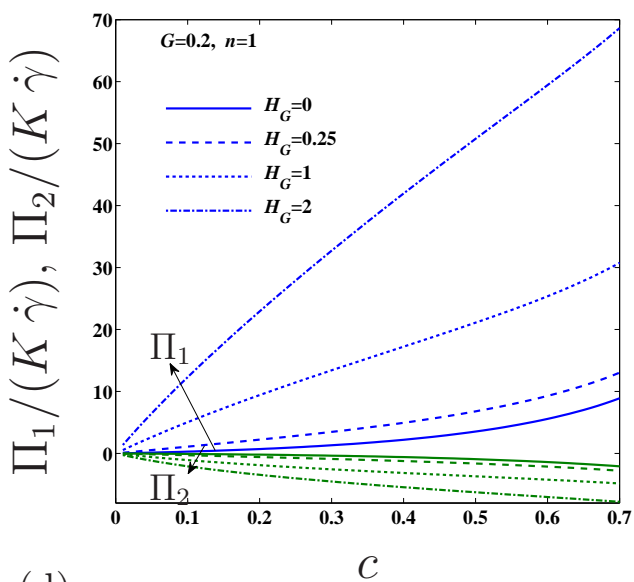

(d)

Figure 4: Variational estimates for the steady-state response of suspensions of initially spherical Gent particles with $G=0.2$ in a Bingham fluid $(n=1)$ for various values of $H_{G}$ under shear flow conditions. The components of $\overline{\boldsymbol{\tau}}^{(1)}$ and $\overline{\boldsymbol{\tau}}^{(2)}$ are given relative to the fixed coordinate system $\mathbf{E}_{i}$ and the principal axes of the particles, respectively. 
steady-state response of suspensions of Gent particles with $G=0.2$ in a Bingham fluid with varying values of $H_{G}$ under shear flow conditions. We recall that higher values of $H_{G}$ correspond to Bingham fluids with higher yield stresses. As expected, we observe from figures 4 (a) and (b) that, for the case of Newtonian fluids $\left(H_{G}=0\right)$, the SS level of elongation and alignment of the particles with the shear direction increases with the volume fraction of particles. However, for the case of Bingham fluids with large enough values of $H_{G}$, the SS shape and orientation of the particles are seen to be weakly affected by the volume fraction. This can be explained by noting that at higher values of $H_{G}=\tau_{0} / \mu$ (corresponding to larger $\tau_{0}$ at a fixed $\mu)$, the suspending Bingham fluid behaves like a very stiff material, so that the deformation in the particle is negligibly affected by increasing the concentration of the particles. Next, figures 4(c) and (d) show the corresponding estimates for the macroscopic shear and normal stress differences, respectively. In particular, we observe from figure $4(\mathrm{c})$ that, at low values of $H_{G}$, the macroscopic shear stress is increasing with the volume fraction of particles, but this trend is found to be reversed for higher values of $H_{G}$. This is, once again, due to the fact that, at higher values of $H_{G}$, the fluid matrix behaves much stiffer than the particles, so that the addition of more particles makes the suspension softer in the shear direction. On the other hand, we observe from figure 4(d) that the (macroscopic) normal stress differences exhibit a more pronounced increase with the volume fraction as $H_{G}$ increases.

\subsection{Power-law fluids}

In this subsection, we present results for the case of suspensions of elastic (Gent) particles in a power-law fluid (i.e., $H=0$ ). Figure 5 shows variational estimates for the time-dependent response of suspensions of Gent particles with $G=0.2$ in a power-law fluid under shear flow. Results are shown for a fixed volume fraction $(c=0.2)$ and various values of the power-law index $n$. Although our model can be used for general values of $n>0$, here, we confine our results to the case of $0<n \leq 1$. This is because, as mentioned earlier, the power-law fluids with $n<1$ exhibit shearthinning behavior which is more common than shear-thickening behavior, and often 


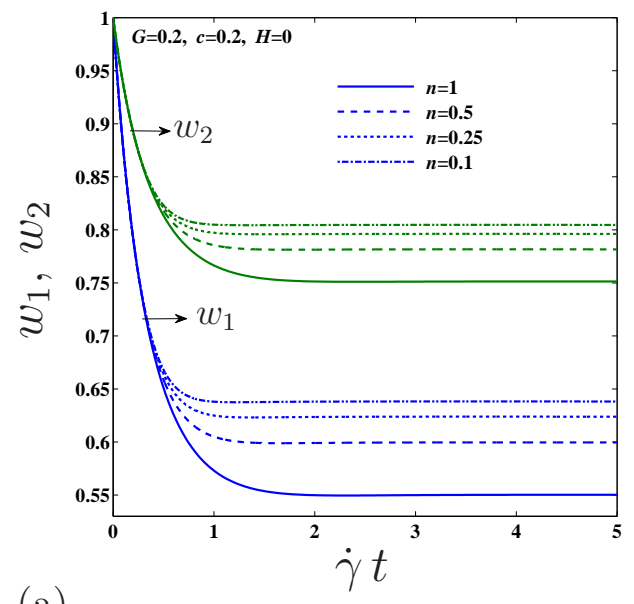

(a)

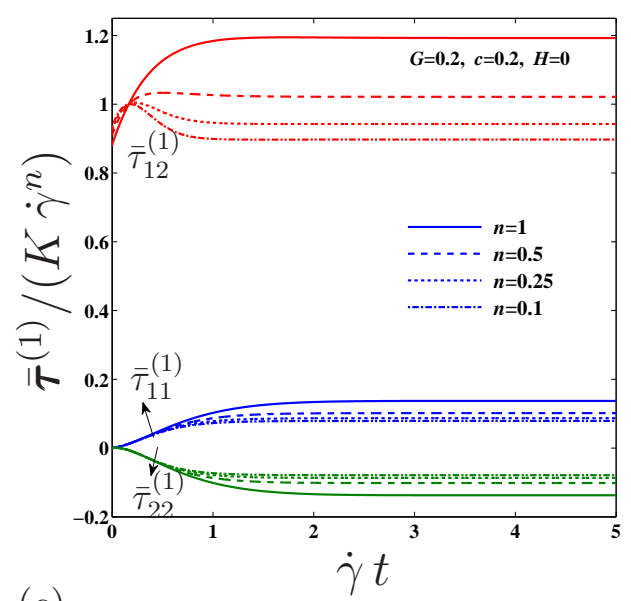

(c)

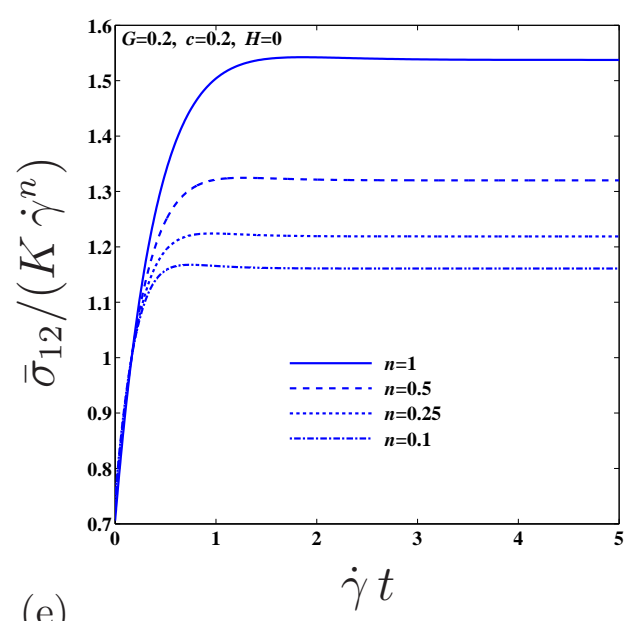

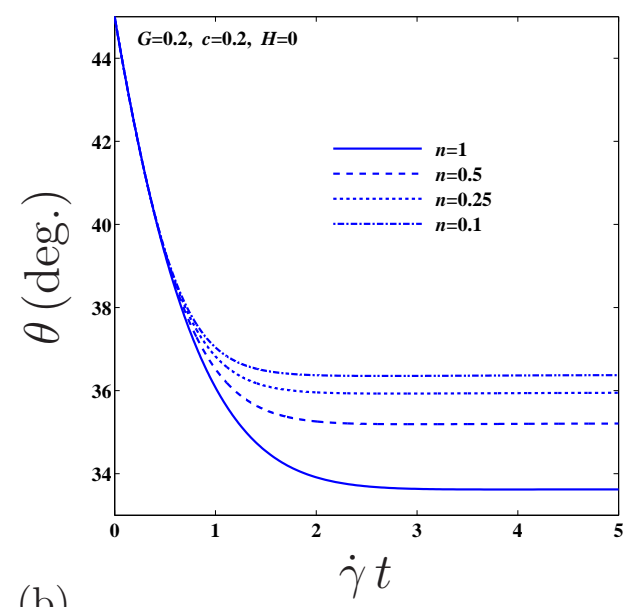

(b)

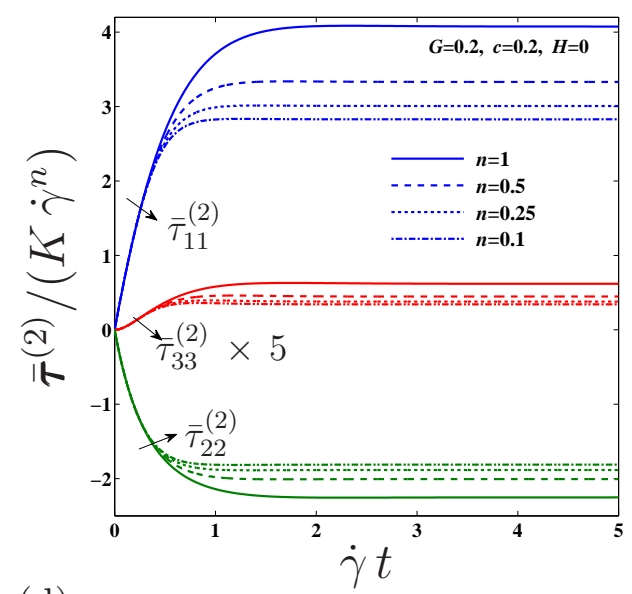

(d)

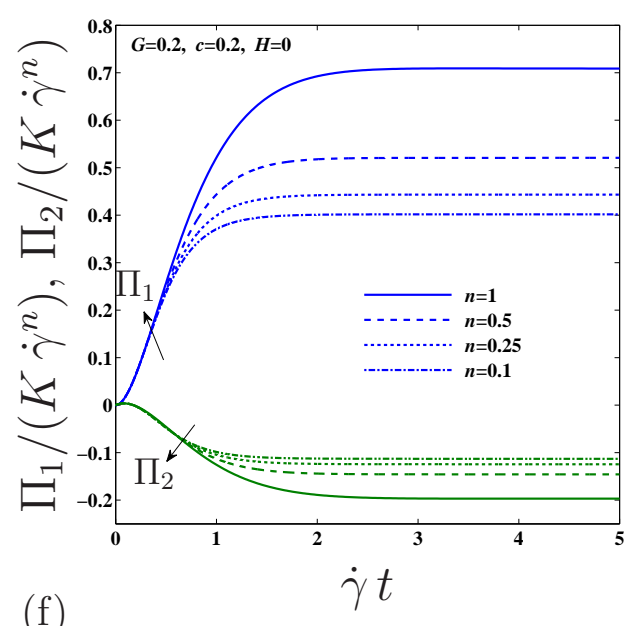

(f)

Figure 5: Variational estimates for the time-dependent response of suspensions of initially spherical Gent particles with $G=0.2$ in a power-law fluid $(H=0)$ for various values of $n$ under shear flow conditions. The components of $\overline{\boldsymbol{\tau}}^{(1)}$ and $\overline{\boldsymbol{\tau}}^{(2)}$ are given relative to the fixed coordinate system $\mathbf{E}_{i}$ and the principal axes of the particles, respectively. 
seen in polymer solutions, molten polymers, and complex fluids such as blood, paint, and ketchup. The evolutions of $w_{1}, w_{2}$ and $\theta$ in figures 5 (a) and (b) show that over short times, say less than $3 \mathrm{~s}$ for $\dot{\gamma}=1$, the particles suspended in a power-law fluid deform and rotate rapidly and then they reach a steady state. Interestingly, we further observe from these figures that, for the value of $G=0.2$, the elastic particles exhibit smaller deformations, as well as less alignments with the shear direction at the steady state, as the value of the index $n$ decreases. This is linked to the fact that a suspending power-law fluid with a smaller value of $n$ exhibits a greater degree of shear-thinning, inducing smaller steady-state values of stress in the fluid and particles, as observed from figures 5 (c) and (d). Consistently, we observe from figures 5(e) and (f) that the corresponding macroscopic shear stress as well as normal stress differences reach a smaller value at the steady state with decreasing $n$. Moreover, we observe from figure 5(e) that the normalized (macroscopic) shear stress increases with the duration of shearing up (before reaching a steady state), which is the characteristic of rheopectic fluids. It is also interesting to mention that the effect of decreasing the power-index $n$ on the macroscopic shear stress of the suspensions of elastic particles in a power-law fluid at a fixed volume fraction of the particles is (qualitatively) very similar to the effect of increasing the volume fraction of elastic particles on the macroscopic shear stress of the suspensions of elastic particles in a Newtonian fluid $(n=1)$, which can be observed from figure 5(c) in [19]. This can be explained by noting that both effects cause a shear-thinning behavior in the respective suspensions.

Next, similar to the case of Bingham fluids discussed in the previous subsection, we investigate the steady-state response of the suspensions of Gent particles in a power-law fluid in simple shear. Figure 6 shows variational estimates for the steadystate behavior of the particle, as well as for the macroscopic stresses, as a function of $G$. The results are given for a fixed volume fraction $(c=0.2)$ and several values of $n$. Similar to the results in figures $3(\mathrm{a})$ and (b) for the case of Bingham fluids, we observe from figure 6 (a) and (b) that, as $G$ increases, the elastic particles reach larger elongations, as well as closer alignments with the shear direction at the steady 


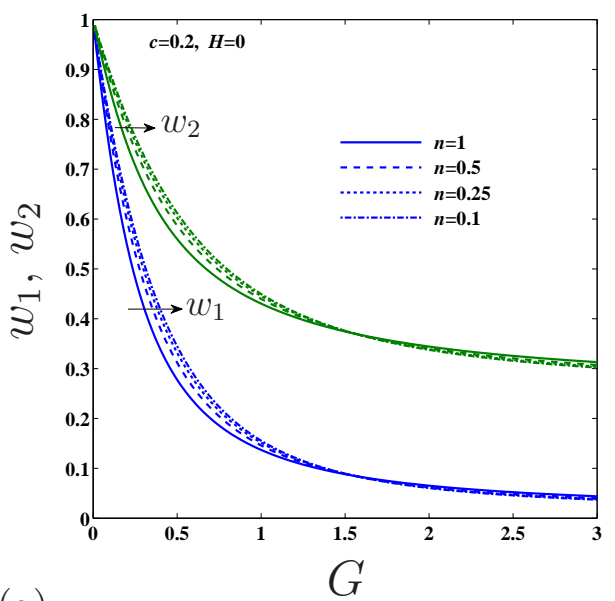

(a)

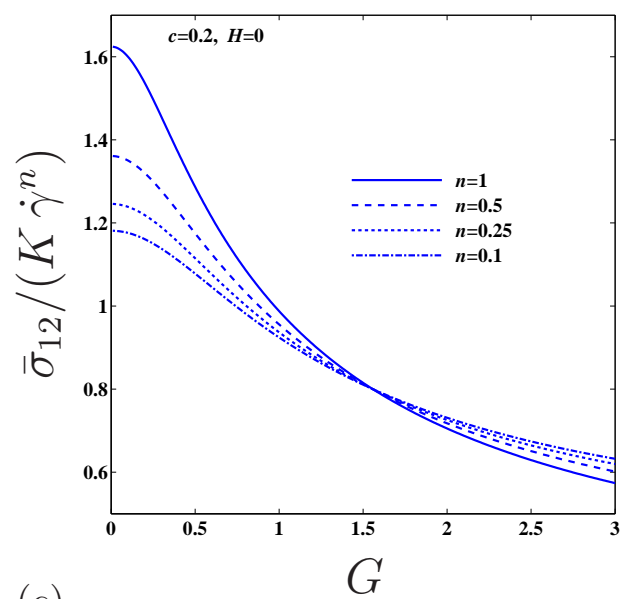

(c)

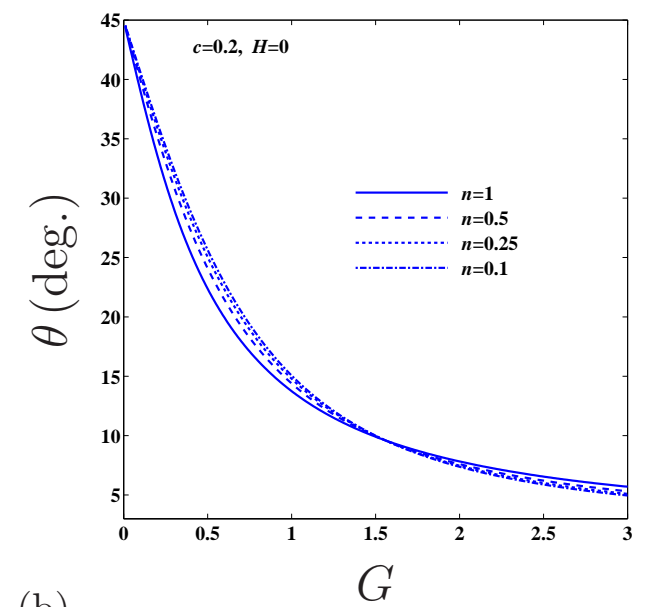

(b)

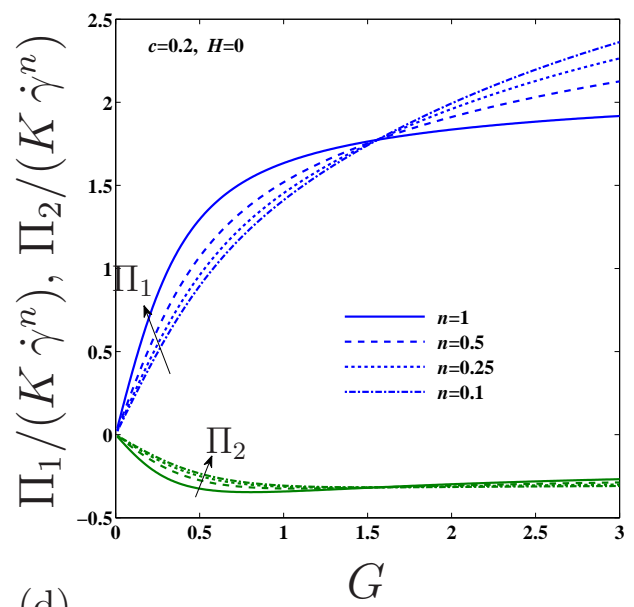

(d)

Figure 6: Variational estimates for the steady-state response of suspensions of initially spherical Gent particles with $G=0.2$ in a power-law fluid $(H=0)$ for various values of $n$ under shear flow conditions. The components of $\overline{\boldsymbol{\tau}}^{(1)}$ and $\overline{\boldsymbol{\tau}}^{(2)}$ are given relative to the fixed coordinate system $\mathbf{E}_{i}$ and the principal axes of the particles, respectively. 
state. However, this behavior of the particles is seen to be weakly affected by the value of $n$. Next, figures 6(c) and (d) show the corresponding estimates for the macroscopic shear and normal stress differences, respectively. We can see from figure 6 (c) that the suspensions of elastic particles in power-law fluids exhibit a shearthinning effect which is stronger for larger values of $n(\leq 1)$. In this connection, we note that, the shear-thinning effect observed in these suspensions (and, in general, in suspensions of deformable particles in HB fluids with $n<1$ ) is derived from two sources: the shear-thinning characteristic of the fluid matrix and the deformation of the particles subjected to shear flows. Another related observation from figure 6(c) is that the (normalized) macroscopic shear stress for the case of Newtonian fluids $(n=1)$ becomes unity at approximately $G=0.88$. This value of $G$, at which the suspended elastic particles do not change the effective shear stress of the host liquid, was first reported in [16] for the case of dilute concentration of elastic particle in Newtonian fluids, and was shown later in [19] to be fairly insensitive to the value of the volume fraction of elastic particles. However, it is evident from figure 6(c), this value (denoted by $G_{N}$ in earlier work), decreases with decreasing values of $n$. Finally, we note that, when $G \rightarrow 0$, the values of the shear stress in figure $6(\mathrm{c})$ match the corresponding values predicted by relation (73).

Lastly, in this subsection, figure 7 shows the effect of particle concentration $c$ on the variational estimates for the SS value of the relevant variables in suspensions of Gent particles with $G=0.2$ in a power-law fluid with different values of $n$ under shear flow conditions. Consistent with earlier comments in the context of figure 4 for the case of Bingham fluids, we observe from figures 7 (a) and (b) that the elastic particles in a power-law fluid (with values of $n>0.5$ ) reach larger elongations and closer alignments with the shear direction with increasing particle concentration $c$. However, it is interesting to observe that, for very small values of $n$, the particle elongation exhibits an opposite behavior and the particle orientation shows only a slight additional alignment with the shear direction as $c$ increases from 0 to 0.7 . As explained in the context of figure 5, this is due to the fact that a power-law suspending fluid with a lower index $n$ exhibits a stronger shear-thinning behavior, leading to 


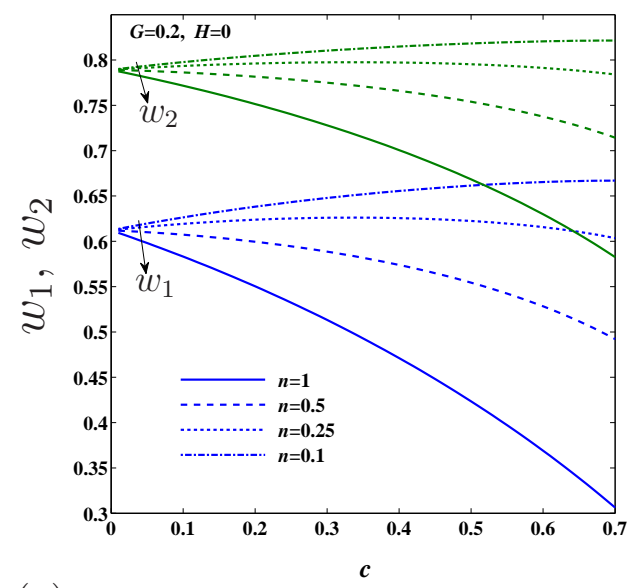

(a)

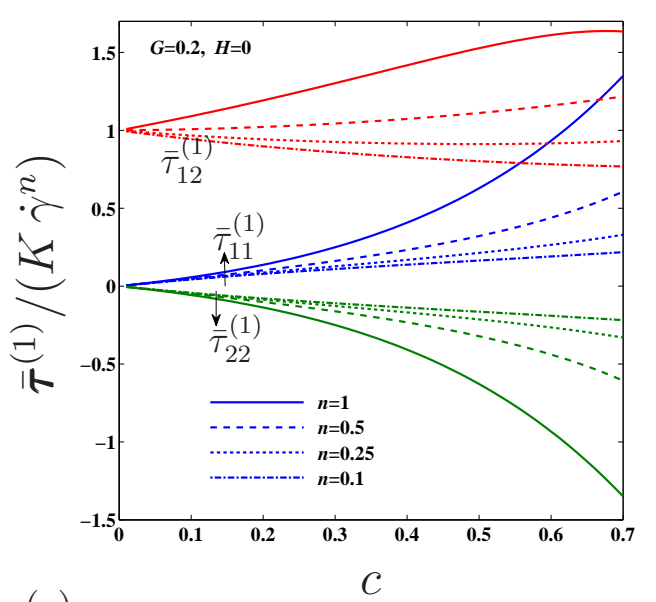

(c)

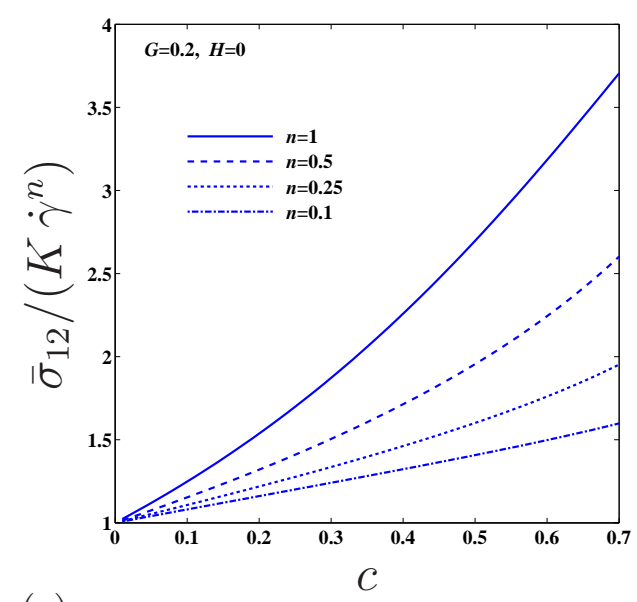

(e)

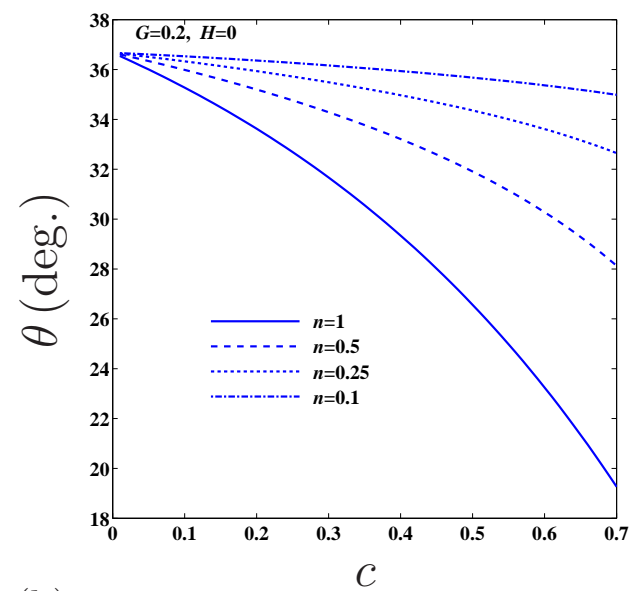

(b)

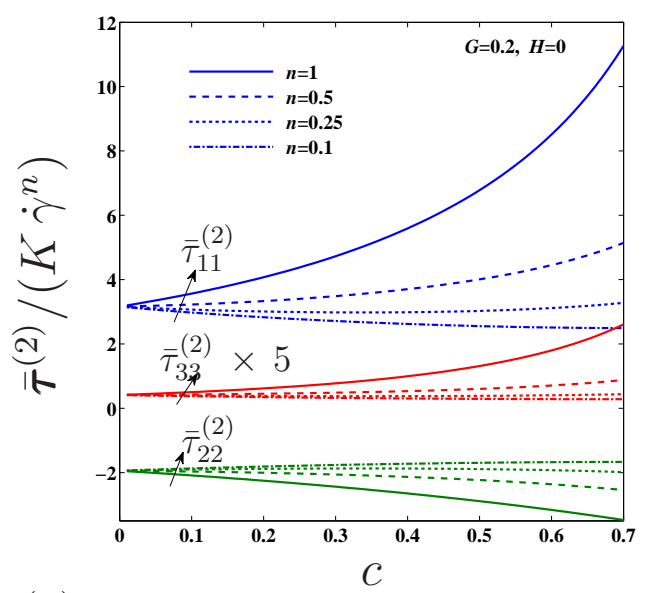

(d)

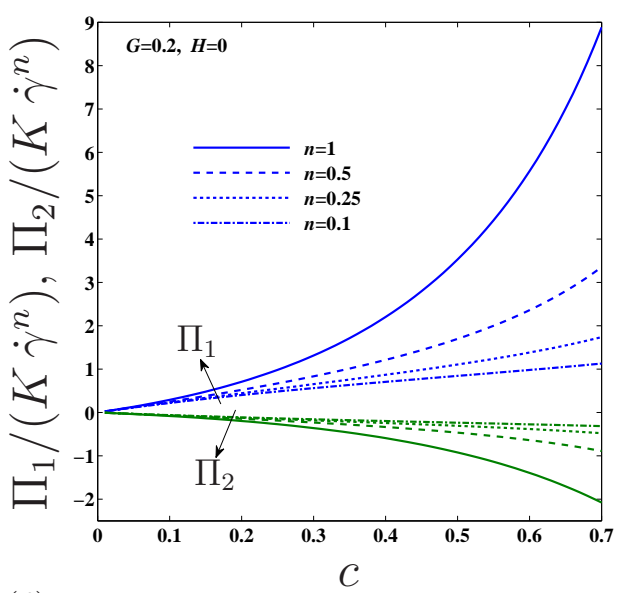

(f)

Figure 7: Variational estimates for the steady-state response of suspensions of initially spherical Gent particles in a power-law fluid $(H=0)$ for various values of $n$ under shear flow conditions. The components of $\overline{\boldsymbol{\tau}}^{(1)}$ and $\overline{\boldsymbol{\tau}}^{(2)}$ are given relative to the fixed coordinate system $\mathbf{E}_{i}$ and the principal axes of the particles, respectively. 
smaller SS values of stress in the fluid and particle phases, as observed from figures 7 (c) and (d). Consequently, we observe from figures 5 (e) and (f) that the effect of the particle concentration on the corresponding estimates for the macroscopic shear and normal stress differences becomes less significant as the suspending fluids become more shear-thinning (i.e., as $n$ decreases).

\subsection{Herschel-Bulkley fluids}

Finally, in this subsection, we present results for the case of suspensions of elastic (Gent) particles in a general Herschel-Bulkley fluid. Figure 8 presents variational estimates for the time-dependent behavior of suspensions of purely elastic (Gent) particles in a Herschel-Bulkley fluid ( $H=1$ and $n=0.5)$ subjected to the shear flow conditions (63). Results are shown for a fixed volume fraction $(c=0.2)$ and different values of $G=K \dot{\gamma}^{n} / \mu$. Similar to our observation for the case of Bingham and powerlaw fluids (discussed in the context of figures 2 and 5, respectively), we observe from this figure that the evolution of particle shape and orientation, the average of stress in the matrix and particle phases, and the macroscopic stresses exhibit a transient behavior which leads into a steady-state regime. In particular, beyond the effective yield stress threshold, the time-dependent macroscopic behavior of the present suspensions qualitatively resemble the behavior of a Maxwellian viscoelastic

fluid, where the stress that is induced by application of a constant shear strain rate relaxes over time. However, a pronounced "overshoot" is observed in the behavior of these suspensions, which is due to the direct influence of the shape dynamics of the suspended soft particles on the macroscopic behavior of these suspensions, as will be discussed shortly. The evolutions of $w_{1}, w_{2}$ and $\theta$ in figures 8 (a) and (b) indicate that, as $G$ increases, the elastic particles exhibit larger elongations, as well as closer alignment with the shear direction. In addition, we observe from these figures that, at a constant strain rate $\dot{\gamma}$, it would generally take longer for the particles with a higher $G$ (corresponding to softer particles) to reach a steady state. In this connection, we, however, observe from these figures that the particle rotations reach a steady state progressively later than the particle elongation in the plane of shear (characterized 

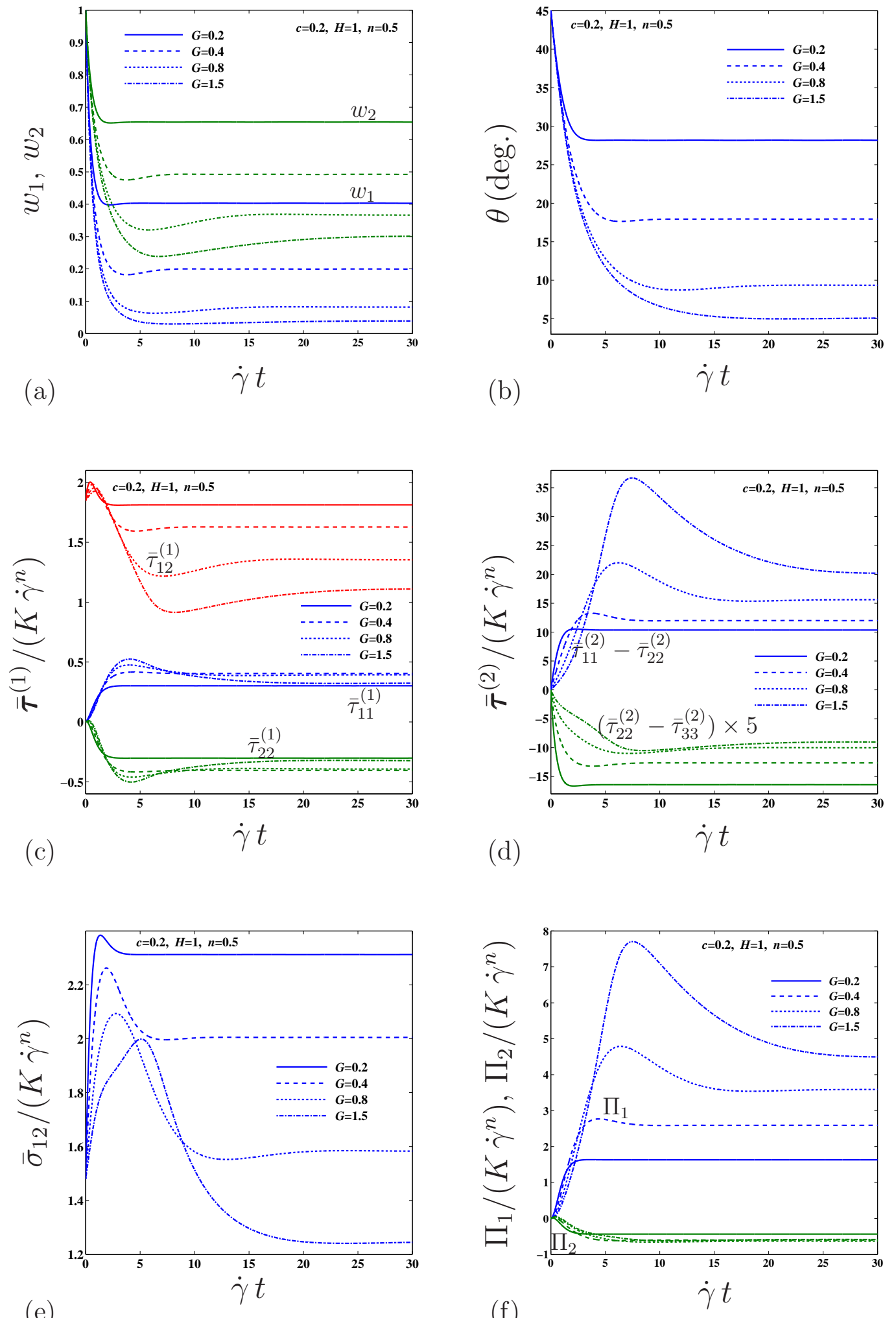

(e)

Figure 8: Variational estimates for the time-dependent response of suspensions of initially spherical Gent particles with $c=0.2$ and various values of $G$ in a Herschel-Bulkley fluid ( $H=1$ and $n=0.5$ ) under shear flow conditions. Higher values of $G$ correspond to softer particles. The components of $\overline{\boldsymbol{\tau}}^{(1)}$ and $\overline{\boldsymbol{\tau}}^{(2)}$ are given relative to the fixed coordinate system $\mathbf{E}_{i}$ and the principal axes of the particles, respectively. 
by the aspect ratio $w_{1}$ ) with increasing value of $G$. Next, we observe from figure 8 (c) and (d) that the shear stress component $\bar{\tau}_{12}^{(1)}$ in the fluid shows a monotonic reduction in magnitude as $G$ increases, while the normal component $\bar{\tau}_{11}^{(2)}-\bar{\tau}_{22}^{(2)}$ in particle exhibits a rather opposite trend with increasing the value of $G$. This can be explained by noting that, as $G=K \dot{\gamma}^{n} / \mu$ increases (and if we think of this as a decrease in $\mu$ ), the softer particles serve to accommodate more part of the total macroscopic deformation applied to the suspension (by exhibiting larger elongations and closer alignment with the shear direction), so that the smaller and larger stresses are produced in the fluid matrix and the particle, respectively. Moreover, figures 8(e) and (f) show the corresponding estimates for the macroscopic shear and normal stress differences, respectively. An interesting observation from figure 8(e) is presence of an "overshoot", followed by a decay in the macroscopic shear component, as similarly reported in [16] and [19] for suspensions of elastic particles in Newtonian fluids. As explained in these works, this overshoot is linked to the above-mentioned fact that the time interval between the arrest of the particles elongations in the plane of shear and the corresponding arrest of the particles rotations becomes progressively larger as $G$ increases. Finally, an important conclusion from these figures (and similarly from figures 2 and 5) is that a suspension of elastic particles in a Herschel-Bulkley fluid exhibits a time-dependent behavior upon application of a constant strain rate, although there is no time-dependency present at the level of the individual phases.

For completeness, it is of interest to examine the influence of the yield stress of the suspending fluid on the SS behavior of the suspensions of elastic particles in a HB fluid. Figure 9 presents variational estimates for the steady-state response of suspensions of elastic (Gent) particles with $c=0.2$ in a HB fluid in simple shear. Results are shown for various values of $G$, as a function of the non-dimensional parameter $H=\tau_{0} /\left(K \dot{\gamma}^{n}\right)$. We observe from figures 9 (a) and (b) that the elastic particles in a HB fluid exhibit larger stretches as well as closer alignments with the shear direction with increasing values of $H$, which is consistent with the corresponding results presented in figures 2 and 3 for suspensions of elastic particles in a Bingham fluid. This trend, however, is seen to be less pronounced at higher values of $G$ corresponding 


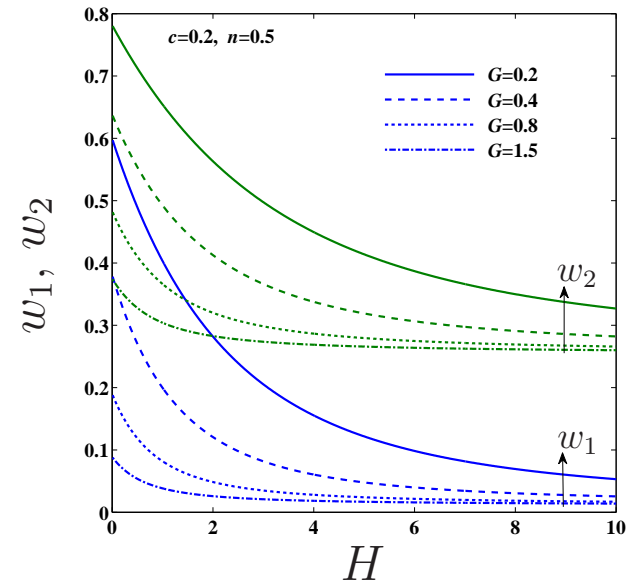

(a)

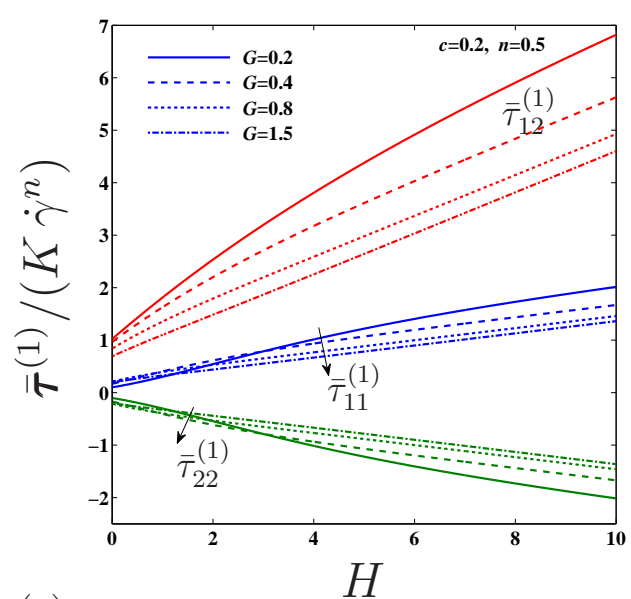

(c)

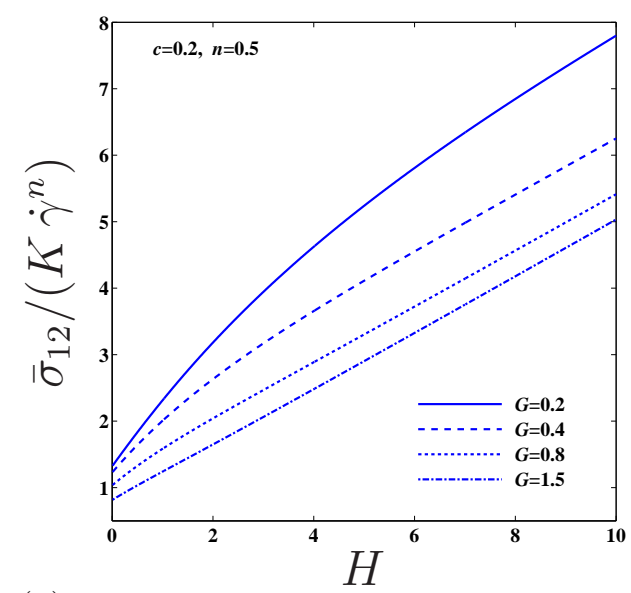

(e)

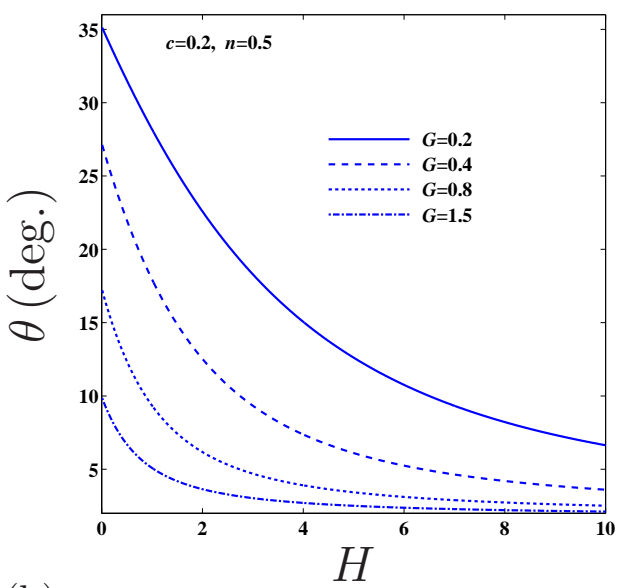

(b)

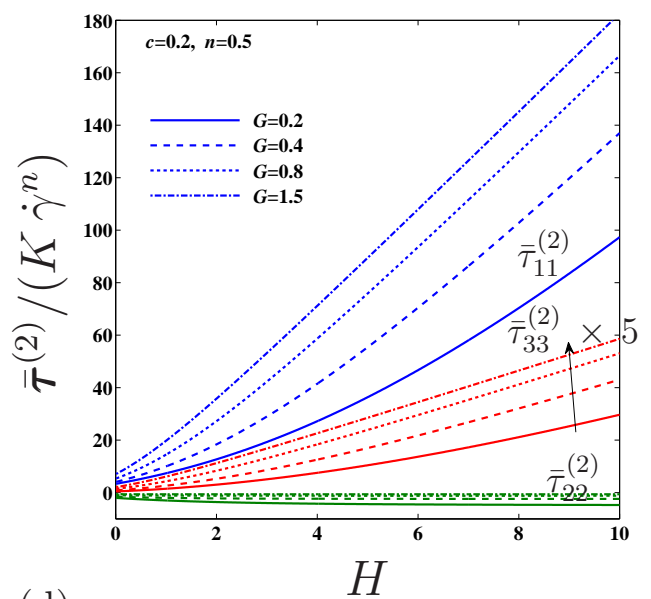

(d)

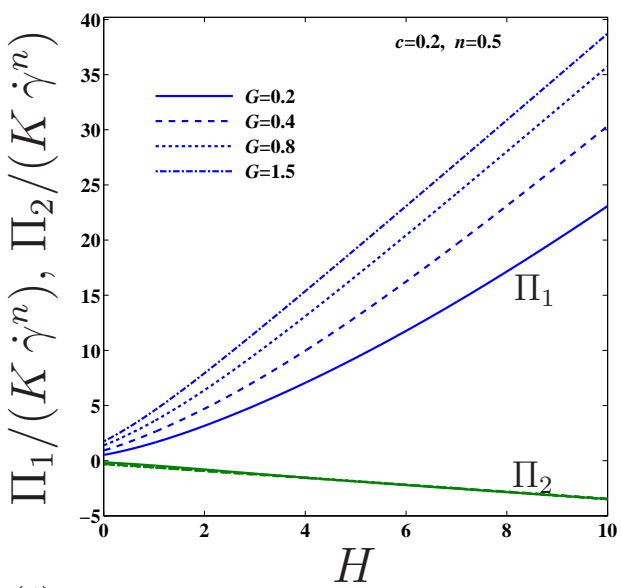

(f)

Figure 9: Variational estimates for the steady-state response of suspensions of initially spherical Gent particles with $c=0.2$ and various values of $G$ in a Herschel--Bulkley fluid $(n=0.5)$ under shear flow conditions. The components of $\overline{\boldsymbol{\tau}}^{(1)}$ and $\overline{\boldsymbol{\tau}}^{(2)}$ are given relative to the fixed coordinate system $\mathbf{E}_{i}$ and the principal axes of the particles, respectively. 


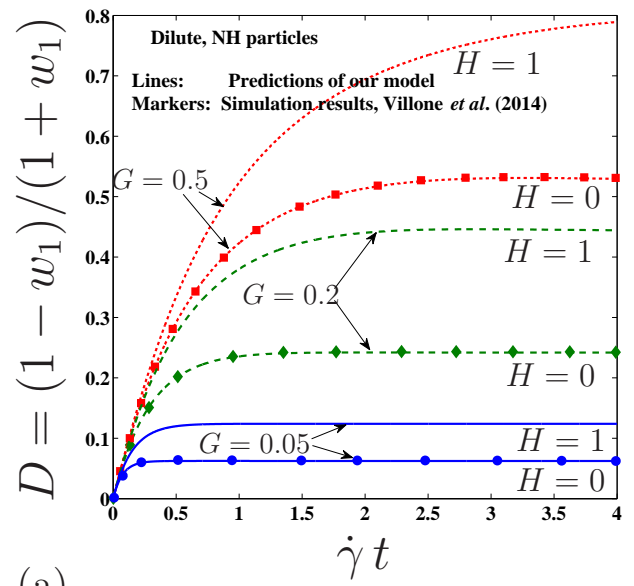

(a)

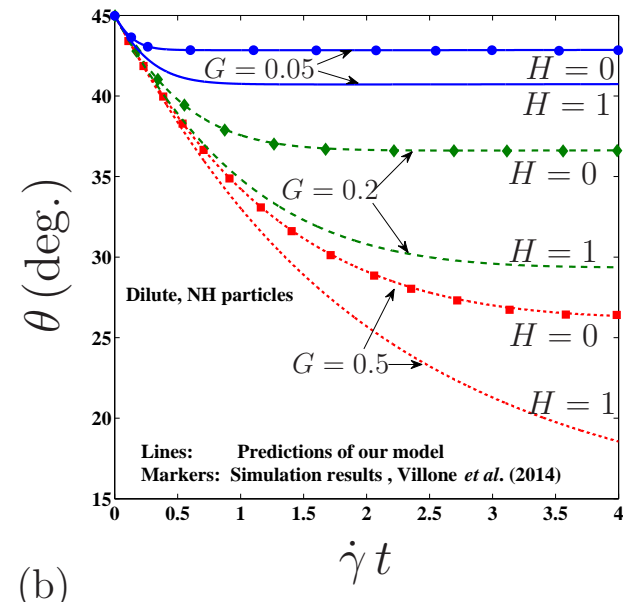

(b)

Figure 10: Comparisons of the predictions of our model with numerical simulation results of Villone et al. [30] for the limiting case of dilute suspensions of initially spherical neo-Hookean (NH) particles in Newtonian fluids $(H=0)$ in shear flow. Plots show comparisons of the time-dependent (a) Taylor deformation parameter, and (b) the rotation angle of the particles. Corresponding predictions of our model for the case of a yield-stress fluid $(H=1)$ are also included for comparison purposes.

to softer particles. Next, we observe from figures 9 (c) and (d) that increasing $H$ leads to an increase in the stress level in both fluid and particle phases for all values of $G$ (which is consistent with the corresponding results in figures 3 for the case of Bingham fluids), while increasing $G$ leads to a reduction and increase in the stress level in the fluid and particle phases, respectively (again, in agreement with the corresponding results in figures 9.) Finally, figures 9(e) and (f) show the corresponding estimates for the macroscopic shear and normal stress differences, respectively. An interesting observation from figures $9(\mathrm{e})$ is that the macroscopic shear stress exhibits fairly linear increase with the parameter $H$ at high enough values of $G$. This behavior of the suspension is qualitatively similar to the corresponding behavior in the suspensions of rigid spherical particles, described by relation (73). This is because, at high enough values of $G$, the SS particle shape and orientation show only a slight dependence on $H$ (see figures 3 (a) and (b)), in such a way that the SS behavior of the suspension resembles that of suspensions of rigid spherical particles.

Also, in figure 10, we compare predictions of our model for dilute suspensions 
of neo-Hookean particles in Newtonian fluids with the corresponding results from recent numerical simulations by Villone et al. [30]. This comparison is limited to the special case of Newtonian fluids, as we were not able to find a complete set of experimental or simulation results for suspensions of soft elastic particles in yield stress fluids to make the appropriate comparisons. However, in this figure, we have also included corresponding predictions of our model for the case of yield stress fluids to highlight the change in the results when the suspending fluid is non-Newtonian. Figures 10 (a) and (b) show the comparisons for the time-dependent behaviour of the Taylor deformation parameter $D=\left(1-w_{1}\right) /\left(1+w_{1}\right)$ and the particle orientation $\theta$, respectively, for three values of $G(G=0.05,0.2$, and 0.5.) We observe excellent agreement between the predictions of our model and the simulation results for the case of Newtonian fluids $(H=0, n=1)$ for both the particle deformation and rotation. In this connection, we recall that, in the limiting case of dilute suspensions of neo-Hookean particles in Newtonian fluids, our model reproduces exactly the corresponding exact estimates of Gao et al. [16] for the time-dependent response of the particle as well as the exact results of Roscoe [13] for steady-response of the particle. Moreover, we observe from these figures that, for the case of a yield stress fluid ( $H=1, n=1)$, the yield stress of the matrix can significantly influence the evolution of the particle shape and orientation. This observation, along with the similar observations discussed earlier in this paper, show the crucial effect of yield stress fluid properties on the rheology of soft particles and demonstrates the need for performing experimental/computational studies on the rheology of suspensions of soft particles in yield stress fluids.

Finally, as mentioned in the Introduction, Chateau and coworkers have recently performed experimental studies on the effective behavior of non-colloidal suspensions of rigid particles [3, 4], as well as bubbles [23, 22] in yield stress fluids. Although our model has been developed to describe the effective response of suspensions of highly-deformable (incompressible) viscoelastic particles, we conducted comparisons with these experimental results for the appropriate limiting cases of our model. As mentioned earlier in this section, our model reduces to the special case of suspensions 


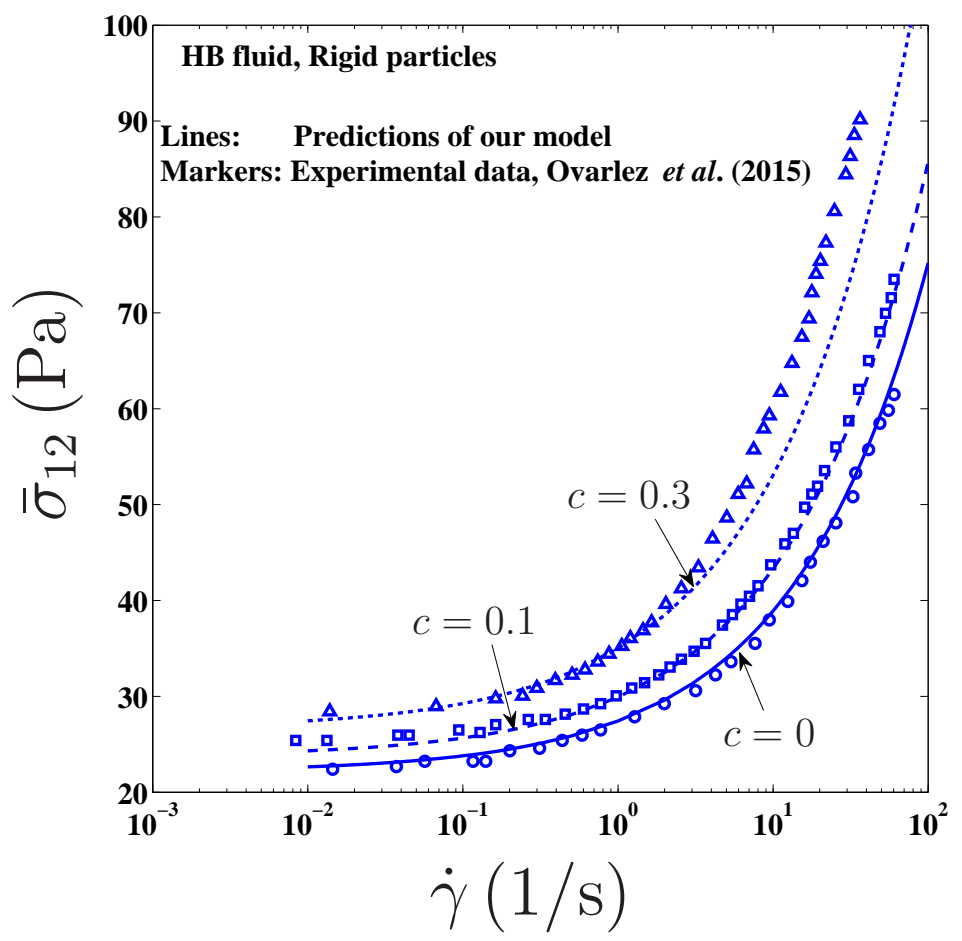

Figure 11: Comparisons of the predictions of our model with experimental data of Ovarlez et al. [4] for the limiting case of non-dilute suspensions of rigid particles in yield stress fluids in shear flow. The experimental data correspond to suspensions of monodisperse spherical beads in an emulsion. The behavior of the matrix was captured by a HB fluid with properties $\tau_{0}=22.1 \mathrm{~Pa}$, $K=5.3 \mathrm{~Pa}^{0.5}{ }^{0.5}$, and $n=0.5$. These properties and experimental data are taken from Figure 7 in [4]. 
of rigid spherical particles in yield stress fluids, in the limiting case of $G \rightarrow 0$. Figure 11 shows comparisons between the predictions of our model for the macroscopic shear stress in non-dilute suspensions of rigid particles in HB fluids (Eq. (73)) with experimental data in [4] for the same quantity in the suspensions of (spherical) glass beads in emulsions. The results are shown for two values of the particle volume fraction ( $c=0.1$, and 0.3$)$, as well as for the pure matrix $(c=0)$ with material

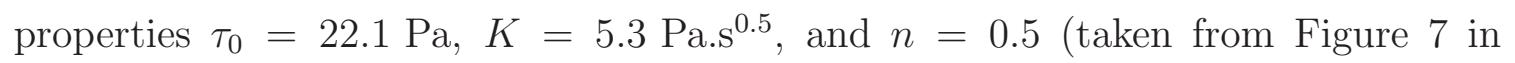
[4].) We observe from this figure that the predictions of our model agree quite well with the experimental data for the case of $c=0.1$ up to high shear rates, and also for the case of $c=0.3$ up to a moderate range of shear rate. Despite this good agreement (at least for the smaller values of $c$ ), it is worth recalling [19] that our model is not expected to provide the most accurate predictions for the case of rigid particles, since it has been assumed (for simplicity) that the shape of (the angular dependence of) the two-point correlation function is instantaneously identical to the shape of the particles. Since for rigid particles the particle shape cannot change, this would mean that the two-point correlations function remains isotropic, which is not expected to be the case for the non-colloidal suspensions considered in this work. In other words, the case of rigid particles is an extreme case for our model where the simplifying assumption for the evolution of the distribution function imposes its strongest limitation on the range of volume fractions for which the model can be expected to provide good estimates for the effective behavior of the suspensions. On the other hand, for deformable particles, the change in shape of the particles is expected to be dominant over the higher-order effects in $c$ associated with the changes in the particle distribution, at least for small to moderate volume fractions $(c<0.3)$, and the model is expected to be more accurate then.

For the case of suspensions of bubbles, we note that our model, in general, can not be specialized to such suspensions, mainly because our model would not account for the effects of surface tension at the bubble/matrix interface in the limiting case of particles approaching bubbles. However, as pointed out in [23] and [22], there are cases of suspensions of bubbles that surface tension forces are negligible and the 
bubbles can be viewed as (freely-deforming) voids inside the fluid matrix. The aforementioned authors achieved such suspensions by introducing big bubbles (radius 1 $\mathrm{mm}$ ) into an emulsions with high stiffness modulus. Therefore, the initial response of such suspensions will be very similar to that of suspensions of deformable particles discussed earlier in this section in the context of Eqs. (65) to (68). In particular, following the homogenization framework of Chateau et al. [10] for suspensions of rigid particles, Ducloué et al. [22] provided variational estimates for the effective yield stress and consistency constant for suspensions of spherical bubbles (with negligible tension force) in yield stress fluids. These estimates, given by relations (9) and (10) in [22], agree exactly with our corresponding estimates in relations $(67)_{1}$ and $(67)_{2}$, respectively. In addition, as shown in figures 4(b) and 7 in [22], these estimates, calculated at $n=1$, provide very good agreement with corresponding experimental results for the effective yield stress and (tangent) viscosity of the aforementioned suspensions of bubbles. Finally, we mention that despite the good agreements between our model predictions and experimental data in the limiting cases, a full investigation of the range of validity of our results remains contingent on the availability of simulation results for suspensions consisting of polydisperse distributions of neo-Hookean particles in HB yield stress fluids.

\section{Conclusions}

In this paper, we have developed a homogenization model to estimate the effective rheological behavior of non-colloidal suspensions of initially spherical, soft particles in yield stress fluids under Stokes flow conditions. More specifically, we considered suspensions of Kelvin-Voigt solid particles in a Herschel-Bulkley fluid undergoing time-dependent, finite deformations when subjected to uniform flows.

Our model can be summarized in four steps: $(i)$ we formulated the problem of finding the effective (instantaneous) response of the suspension in terms of finding the effective potential $\widetilde{W}(\overline{\mathbf{D}})$ of the suspension, as described by relations (22) and (23), (ii) we then made use of the variational principle of Ponte Castañeda [5] to

approximate the potential $\widetilde{W}(\overline{\mathbf{D}})$ of the actual suspension in terms of the effective 
potential $\widetilde{W}_{L}$ of a "linear comparison composite" (LCC) consisting of the same particles distributed randomly in a (fictitious) Newtonian fluid with a suitably-chosen viscosity $\eta^{(1)}$, identified with the optimality conditions (33) and (34), (iii) we recalled the relevant estimates for the LCC from [19], including an estimate for $\widetilde{W}_{L}$, as given by $(40),(i v)$ we combined the solution for the instantaneous behavior of the suspension with the evolution equation for the elastic stress in the particles, as given by (54), and for the average particle shape and orientation, as given by (50) and (51), respectively, to complete the model for the time-dependent response of the suspensions.

We have also used our model to explore in more detail the rheology and particle dynamics in suspensions of elastic Gent particle is HB fluids under shear flow conditions, as described by (63). In the context of this example, we investigated the influence of constitutive properties of the HB fluids and the particles, and the particle volume fraction on the dynamics of the suspended particles, as well as on the macroscopic rheological behavior of the suspension. A general conclusion from the results is that the suspensions exhibit a time-dependent behavior upon application of a constant strain rate, even though the response of the particles and fluids is not time-dependent. Also, we found that the time-dependent behavior of the suspensions qualitatively resemble the behavior of a Maxwellian viscoelastic fluid, where the stress, induced by application of a constant strain rate, relaxes over time. A similar finding has been reported in the context of suspensions of slightly deformable particles in a Newtonian fluid by Goddard [15]. In particular, for Bingham fluid suspensions, our model predicts a non-monotonic time evolution for the shear stress and the first normal stress difference, with a rapid increase up to a peak value and a gentle decrease into a steady-state regime. Also, for the case of power-law fluids (i.e., when $\left.\tau_{0}=0\right)$ with $n \leq 1$, our results indicate that the macroscopic time-dependent shear stress exhibits a rheopectic behavior, where the stress increases with the duration of shearing before reaching a steady state.

The results confirm that steady-state, tank-treading solutions are available for suspensions of Gent particles under shear flow conditions. In particular, the results 
show that the particles suspended in a HB fluid with a higher yield stress $\left(\tau_{0}\right)$ exhibit larger elongations, as well as closer alignments with the shear direction at a steady state, and this behavior is more pronounced for the case of stiffer particles. On the other hand, for the case of power-law fluids, we found that, when the elastic particle are not too soft, they exhibit smaller deformations, as well as less alignments with the shear direction at the steady state, as the value of the index $n$ decreases. Also, for the case of Bingham fluids, we found that, at low values of $\tau_{0}$, SS values of the macroscopic shear stress increase with the volume fraction of the particles, while the opposite is true for higher values of $\tau_{0}$. Furthermore, it has been found that the macroscopic shear stress increases linearly with the yield stress $\tau_{0}$ for the case of very soft particles. This behavior of the suspension is qualitatively similar to the corresponding behavior in the suspensions of rigid spherical particles, described by relation (73).

In summary, our study demonstrates the crucial effect of yield stress and shearthinning properties of the suspending fluid on the average shape dynamics of suspended soft particles, and in turn, on the effective rheological behavior of such suspensions when subjected to simple flows. While by and large most works in the literature have focused on the case of suspensions of rigid particles, our recent studies, including the present work, on the effect of particle deformability open up new frontiers in the micromechanical characterization of the time-dependent behavior of viscoelastic fluids, such as polymeric melts and solutions, as well as complex biological suspensions. The novel insights provided by these studies on the mechanistic link between the shape dynamics of the soft particles and viscoelastic behavior of the suspensions will make possible the design and synthesis of non-colloidal suspensions with novel functional properties for applications in the oil and polymer industries. In addition, in future work, we expect to investigate the effect of non-spherical initial particle shapes [42] to determine the possible existence of steady-state regimes for such suspensions, as well as possible implication for complex biological suspensions. Finally, this work hopefully also serve motivates experimental/numerical studies to investigate the combined effect of particles softness and fluid yield stress on the 
rheology of yield stress fluid suspensions.

\section{Acknowledgements}

This material is based upon work supported by the National Science Foundation under Grant No. CMMI-0969570.

\section{Appendix A. Governing equations for the deformation of spherical parti- cles in a shear flow}

In this appendix, we provide simplified evolution equations for the initially spherical, Gent particles, when subjected to the shear flow conditions defined by (63). Recall that the particles take on general ellipsoidal shapes characterized by the aspect ratios $w_{1}$ and $w_{2}$, but rotate only in the shear plane, the rotation being described by the angle $\theta$. Then, the system of equations (50), (51), and (54) can be shown to simplify to

$$
\left.\begin{array}{rl}
\dot{\bar{\tau}}_{11}^{(2)} & =2\left(\bar{D}_{12}^{(2)}+\bar{W}_{12}^{(2)}\right) \bar{\tau}_{12}^{(2)}+2\left(\bar{\tau}_{11}^{(2)}+\mu\right) \bar{D}_{11}^{(2)}+\left(\bar{\tau}_{11}^{(2)}+\mu\right) \Sigma, \\
\dot{\bar{\tau}}_{22}^{(2)} & =2\left(\bar{D}_{12}^{(2)}-\bar{W}_{12}^{(2)}\right) \bar{\tau}_{12}^{(2)}+2\left(\bar{\tau}_{22}^{(2)}+\mu\right) D_{22}^{(2)}+\left(\bar{\tau}_{22}^{(2)}+\mu\right) \Sigma, \\
\dot{\bar{\tau}}_{33}^{(2)} & =-2\left(\bar{\tau}_{33}^{(2)}+\mu\right)\left(\bar{D}_{11}^{(2)}+\bar{D}_{22}^{(2)}\right)+\left(\bar{\tau}_{33}^{(2)}+\mu\right) \Sigma, \\
\dot{\bar{\tau}}_{12}^{(2)} & =\left(\bar{D}_{12}^{(2)}-\bar{W}_{12}^{(2)}\right) \bar{\tau}_{11}^{(2)}+\left(\bar{D}_{12}^{(2)}+\bar{W}_{12}^{(2)}\right) \bar{\tau}_{22}^{(2)} \\
& +\bar{\tau}_{12}^{(2)}\left(\bar{D}_{11}^{(2)}+\bar{D}_{22}^{(2)}\right)+2 \mu \bar{D}_{12}^{(2)}+\bar{\tau}_{12}^{(2)} \Sigma, \\
\frac{\mathrm{d} w_{1}}{\mathrm{~d} t} & =-2 w_{1} \bar{D}_{11}^{(2)}, \quad \frac{\mathrm{d} w_{2}}{\mathrm{~d} t}=-w_{2} \bar{D}_{11}^{(2)}, \quad \frac{\mathrm{d} \theta}{\mathrm{d} t}=\left(\frac{1+w_{1}^{2}}{1-w_{1}^{2}}\right) \bar{D}_{12}^{(2)}-\bar{W}_{12}^{(2)} \cdot
\end{array}\right\}
$$

where

$$
\Sigma=\left[\left(\bar{\tau}_{11}-\bar{\tau}_{33}\right) \bar{D}_{11}^{(2)}+\left(\bar{\tau}_{22}-\bar{\tau}_{33}\right) \bar{D}_{22}^{(2)}+2 \bar{D}_{12}^{(2)} \bar{\tau}_{12}\right] /\left(J_{m} \mu\right)
$$

The above equations are complemented with two equations (33) and (34) for unknown variables $\eta^{(1)}$ and $\hat{D}_{e q}$. The resulting system of equations, together with relations (41) and (42) for the average strain-rate $\overline{\mathbf{D}}^{(2)}$ and vorticity $\overline{\mathbf{W}}^{(2)}$ in the particles, respectively, can be integrated numerically for the time-dependent solution. The components of the shape tensors $\mathbb{P}$ and $\mathbb{R}$, required for this integration, are available from Appendix D in [19]. 


\section{References}

[1] J. Laven and H.N. Stein. The Einstein coefficient of suspensions in generalized newtonian liquids. J. Rheol., 35(8):1523-1549, 1991.

[2] C. Ancey and H. Jorrot. Yield stress for particle suspensions within a clay dispersion. J. Rheol., 45(2):297-319, 2001.

[3] F. Mahaut, X. Chateau, P. Coussot, and G. Ovarlez. Yield stress and elastic modulus of suspensions of noncolloidal particles in yield stress fluids. J. Rheol., 52:287-313, 2008.

[4] G. Ovarlez, F. Mahaut, S. Deboeuf, N. Lenoir, S. Hormozi, and X. Chateau. Flows of suspensions of particles in yield stress fluids. J. Rheol., 59:1449-1486, 2015 .

[5] P. Ponte Castañeda. The effective mechanical properties of nonlinear isotropic composites. J. Mech. Phys. Solids, 39:45-71, 1991.

[6] M. Zaidman and P. Ponte Castañeda. The finite deformation of nonlinear composite materials. II. Evolution of the microstructure. Int. J. Solids Struct., 33: 1287-1303, 1996.

[7] M. Kailasam and P. Ponte Castañeda. A general constitutive theory for linear and nonlinear particulate media with microstructure evolution. J. Mech. Phys. Solids, 46:427-465, 1998.

[8] P. Ponte Castañeda. On the homogenized behaviour of reinforced and other Bingham composites. Phil. Trans. R. Soc. Lond. A, 361(1806):947-964, 2003.

[9] P. Ponte Castañeda. Second-order homogenization estimates for nonlinear composites incorporating field fluctuations. I. Theory. J. Mech. Phys. Solids, 50: 737-757, 2002. 
[10] X. Chateau, G. Ovarlez, and K.L. Trung. Homogenization approach to the behavior of suspensions of noncolloidal particles in yield stress fluids. J. Rheol., 52:489-506, 2008.

[11] J. Férec, A. Perrot, and G. Ausias. Toward modeling anisotropic yield stress and consistency induced by fiber in fiber-reinforced viscoplastic fluids. J. NonNewton. Fluid, 220:69-76, 2015.

[12] A.G. Gibson and S. Toll. Mechanics of the squeeze flow of planar fibre suspensions. J. Non-Newton. Fluid, 82(1):1-24, 1999.

[13] R. Roscoe. On the rheology of a suspension of viscoelastic spheres in a viscous liquid. J. Fluid Mech., 28:273-293, 1967.

[14] J.D. Goddard and C. Miller. Nonlinear effects in the rheology of dilute suspensions. J. Fluid Mech., 28:657-673, 1967.

[15] J.D. Goddard. An elastohydrodynamic theory for the rheology of concentrated suspensions of deformable particles. J. Non-Newton. Fluid, 2(2):169-189, 1977.

[16] T. Gao, H.H. Hu, and P. Ponte Castañeda. Rheology of a suspension of elastic particles in a viscous shear flow. J. Fluid Mech., 687:209-237, 2011.

[17] T. Gao, H.H. Hu, and P. Ponte Castañeda. Shape dynamics and rheology of soft elastic particles in a shear flow. Phys. Rev. Let., 108:058302, 2012.

[18] T. Gao, H.H. Hu, and P. Ponte Castañeda. Dynamics and rheology of elastic particles in an extensional flow. J. Fluid Mech., 715:573-596, 2013.

[19] R. Avazmohammadi and P. Ponte Castañeda. The rheology of non-dilute dispersions of highly deformable viscoelastic particles in newtonian fluids. J. Fluid Mech., 763:386-432, 2015.

[20] Z. Hashin and S. Shtrikman. A variational approach to the theory of the elastic behavior of multiphase materials. J. Mech. Phys. Solids, 11:127-140, 1963. 
[21] J.R. Willis. Bounds and self-consistent estimates for the overall moduli of anisotropic composites. J. Mech. Phys. Solids, 25:185-202, 1977.

[22] L. Ducloué, O. Pitois, J. Goyon, X. Chateau, and G. Ovarlez. Rheological behaviour of suspensions of bubbles in yield stress fluids. J. Non-Newton. Fluid, 215:31-39, 2015.

[23] L. Ducloué, O. Pitois, J. Goyon, X. Chateau, and G. Ovarlez. Coupling of elasticity to capillarity in soft aerated materials. Soft Matter, 10(28):5093-5098, 2014 .

[24] D.D. Joseph. Fluid dynamics of viscoelastic liquids. Springer-Verlag New York, 1990.

[25] A.N. Gent. A new constitutive relation for rubber. Rubber Chem. Tech., 69: 59-61, 1996.

[26] R.W. Ogden, G. Saccomandi, and I. Sgura. Fitting hyperelastic models to experimental data. Comput. Mech., 34:484-502, 2004.

[27] W.H. Herschel and R. Bulkley. Konsistenzmessungen von gummibenzollösungen. Kolloid-Zeitschrift, 39(4):291-300, 1926.

[28] A.N. Alexandrou, T.M. McGilvreay, and G. Burgos. Steady herschel-bulkley fluid flow in three-dimensional expansions. J. Non-Newton. Fluid Mech., 100 (1):77-96, 2001.

[29] J.R. Clausen, D.A. Reasor, and C.K. Aidun. The rheology and microstructure of concentrated non-colloidal suspensions of deformable capsules. J. Fluid Mech., 685:202-234, 2011.

[30] M.M. Villone, M.A. Hulsen, P.D. Anderson, and P.L. Maffettone. Simulations of deformable systems in fluids under shear flow using an arbitrary lagrangian eulerian technique. Comput. Fluids, 90:88-100, 2014. 
[31] M.M. Villone, F. Greco, M.A. Hulsen, and P.L. Maffettone. Simulations of an elastic particle in newtonian and viscoelastic fluids subjected to confined shear flow. J. Non-Newton. Fluid Mech., 2014.

[32] P. Ponte Castañeda and P. Suquet. Nonlinear composites. Adv. Appl. Mech., 34(998):171-302, 1998.

[33] J.B. Keller, L.A. Rubenfeld, and J.E. Molyneux. Extremum principles for slow viscous flows with applications to suspensions. J. Fluid Mech., 30(01):97-125, 1967.

[34] J.R. Willis. Variational and related methods for the overall properties of composites. Adv. Appl. Mech., 21:1-78, 1981.

[35] M.I. Idiart and P. Ponte Castañeda. Field statistics in nonlinear composites. I. Theory. Proc. R. Soc. Lond. A, 463:183-202, 2007.

[36] P. Ponte Castañeda and J.R. Willis. The effect of spatial distribution on the effective behavior of composite materials and cracked media. J. Mech. Phys. Solids, 43:1919-1951, 1995.

[37] P. Ponte Castañeda. New variational principles in plasticity and their application to composite materials. J. Mech. Phys. Solids, 40:1757-1788, 1992.

[38] M.I. Idiart and P. Ponte Castañeda. Variational linear comparison bounds for nonlinear composites with anisotropic phases. i. general results. 463(2080):907924, 2007.

[39] B.A. Bilby and M.L. Kolbuszewski. The finite deformation of an inhomogeneity in two-dimensional slow viscous incompressible flow. Proc. R. Soc. Lond. A., 355:335-353, 1977.

[40] R.W. Ogden. Non-linear Elastic Deformations. Dover, New York, 1997. 
[41] N. Aravas and P. Ponte Castañeda. Numerical methods for porous metals with deformation-induced anisotropy. Comput. Meth. Appl. Mech. Engng., 193:37673805, 2004.

[42] M.M. Villone, G. d'Avino, M.A. Hulsen, and P.L. Maffettone. Dynamics of prolate spheroidal elastic particles in confined shear flow. Phys. Rev. E, 92(6): 062303, 2015. 